\title{
Constraints on cosmic opacity and beyond the standard model physics from cosmological distance measurements
}

\author{
Anastasios Avgoustidis, ${ }^{a}$ Clare Burrage, ${ }^{b}$ Javier Redondo, ${ }^{c}$ \\ Licia Verde ${ }^{d}$ and Raul Jimenez ${ }^{d}$ \\ ${ }^{a}$ Centre for Theoretical Cosmology, DAMTP, CMS, \\ Wilberforce Road, Cambridge CB3 0WA, U.K. \\ ${ }^{b}$ Deutsches Elektronen Synchrotron DESY, \\ Notkestrasse 85, D-22607 Hamburg, Germany \\ ${ }^{c}$ Max Planck Institut für Physik, \\ Föhringer Ring 6, D-80805, Munich, Germany \\ ${ }^{d}$ ICREA \& Institute for Sciences of the Cosmos (ICC), University of Barcelona, \\ IEEC, Barcelona 08028, Spain \\ E-mail: a.avgoustidis@damtp.cam.ac.uk,clare.burrage@desy.de, \\ redondo@mppmu.mpg.de, liciaverde@icc.ub.edu, raul.jimenez@icc.ub.edu
}

Received April 21, 2010

Accepted October 4, 2010

Published October 22, 2010

\begin{abstract}
We update constraints on cosmic opacity by combining recent SN Type Ia data with the latest measurements of the Hubble expansion at redshifts between 0 and 2. The new constraint on the parameter $\epsilon$ parametrising deviations from the luminosity-angular diameter distance relation $\left(d_{L}=d_{A}(1+z)^{2+\epsilon}\right)$, is $\epsilon=-0.04_{-0.07}^{+0.08}(2-\sigma)$. For the redshift range between 0.2 and 0.35 this corresponds to an opacity $\Delta \tau<0.012$ (95\% C.L.), a factor of 2 stronger than the previous constraint. Various models of beyond the standard model physics that predict violation of photon number conservation contribute to the opacity and can be equally constrained. In this paper we put new limits on axion-like particles, including chameleons, and mini-charged particles.
\end{abstract}

Keywords: supernova type Ia - standard candles, axions, cosmological parameters from LSS, dark energy theory

ArXiv ePrint: 1004.2053 


\section{Contents}

1 Introduction $\quad 1$

2 An update on cosmic opacity constraints 2

3 Axion-like particles and chameleons $\quad 5$

$\begin{array}{ll}3.1 & \text { Modelling the effects of ALPs }\end{array}$

3.2 Weak mixing 9

$\begin{array}{lll}3.3 & \text { Strong mixing } & 10\end{array}$

$\begin{array}{lll}3.4 & \text { Numerical results } & 10\end{array}$

$\begin{array}{llr}3.5 & \text { Constraints } & 12\end{array}$

$\begin{array}{lll}\text { 3.5.1 Axion-like-particles } & 12\end{array}$

$\begin{array}{lll}\text { 3.5.2 Chameleons } & 14\end{array}$

4 Mini-charged particles/hidden photons $\quad 15$

5 Forecasts for future baryon acoustic oscillations and Supernovae surveys 18

6 Conclusions $\quad 21$

A Summary of key formulae $\quad 25$

\section{Introduction}

Cosmological observations provide constraints on different distance measures: luminosity distance (as provided e.g., by supernovae), angular diameter distance (as provided e.g., by baryon acoustic oscillations) and even on the expansion rate or the Hubble parameter as a function of redshift $z$. Both luminosity distance and angular diameter distance are functions of the Hubble parameter. While combining these measurements helps to break parameter degeneracies and constrain cosmological parameters, comparing them helps to constrain possible deviations from the assumptions underlying the standard cosmological model (e.g. isotropy), or to directly constrain physics beyond the standard model of particle physics (e.g. couplings of photons to scalar or pseudo-scalar matter).

The Etherington relation [1] implies that, in a cosmology based on a metric theory of gravity, distance measures are unique: the luminosity distance is $(1+z)^{2}$ times the angular diameter distance. This is valid in any cosmological background where photons travel on null geodesics and where, crucially, photon number is conserved.

There are several scenarios in which the Etherington relation would be violated: for instance we can have deviations from a metric theory of gravity, photons not traveling along unique null geodesics, variations of fundamental constants, etc. In this paper we want to restrict our attention on violations of the Etherington relation arising from the violation of photon conservation.

A change in the photon flux during propagation towards the Earth will affect the Supernovae $(\mathrm{SNe})$ luminosity distance measures but not the determinations of the angular diameter distance. Photon conservation can be violated by simple astrophysical effects or by exotic 
physics. Amongst the former we find, for instance, attenuation due to interstellar dust, gas and/or plasmas. Most known sources of attenuation are expected to be clustered and can be typically constrained down to the $0.1 \%$ level $[2,3]$. Unclustered sources of attenuation are however much more difficult to constrain. For example, grey dust [4] has been invoked to explain the observed dimming of Type Ia Supernovae without resorting to cosmic acceleration.

More exotic sources of photon conservation violation involve a coupling of photons to particles beyond the standard model of particle physics. Such couplings would mean that, while passing through the intergalactic medium, a photon could disappear -or even (re)appear!- interacting with such exotic particles, modifying the apparent luminosity of sources. Here we consider the mixing of photons with scalars, known as axion-like particles, and the possibility of mini-charged particles which have a tiny, and unquantised electric charge. A recent review [5] highlights the rich phenomenology of these weakly-interactingsub-eV-particles (WISPs), whose effects have been searched for in a number of laboratory experiments and astronomical observations. In particular, the implications of these particles on the SN luminosity have been described in a number of publications [6-10].

One of the most interesting features of these models is that the exotic opacity involved could in principle "mimic" the value of a non-zero cosmological constant inferred from $\mathrm{SNe}$ measurements. However, this possibility can already be excluded (at least in the simplest WISP models) by the absence of distortions in the CMB or the spectra of quasars for axionlike-particles, and by arguments of stellar evolution in the case of mini-charged particles.

In this paper we use improved bounds on cosmic opacity to further constrain the existence of exotic particles which can couple to the photon. The rest of the paper is organised as follows. In section 2 we update constraints on transparency from the latest available data. In section 3 we discuss the implications of this for axion-like particles and chameleons, and in section 4 we consider mini-charged particles. We then forecast, in section 5 , how the constraints will improve with distance measures from future, planned and proposed, surveys. We conclude in section 6 . Sections 3 and 4 discuss in detail the motivation, modelling and regime of applicability of the beyond the standard model physics we consider. Readers with a more focused interest on cosmology may concentrate on the beginning of section 3 , subsections 3.4, 3.5 and figures 2, 3, 4, 5, 6. Appendix A summarises the cosmologically-relevant results of sections 3 and 4 .

Precursors of this paper can be found in [11-15].

\section{$2 \quad$ An update on cosmic opacity constraints}

In reference [15], the authors use Type Ia SN brightness data (namely the SCP Union 2008 compilation [16]) in combination with measurements of cosmic expansion $H(z)$ from differential aging of luminous red galaxies (LRGs) $[17,18]$ to obtain constraints on non-trivial opacity, at cosmological scales. The basic idea is to study possible violations from the "Etherington relation" [1], the distance duality between luminosity distance, $d_{L}$, and angular diameter distance, $d_{A}$ :

$$
d_{L}(z)=(1+z)^{2} d_{A}(z) .
$$

This identity depends only on photon number conservation and local Lorentz invariance. It holds for general metric theories of gravity, where photons travel along unique null geodesics. Since Lorentz violation is strongly constrained for the low energies corresponding to optical observations [19], the study of possible violations of eq. (2.1) through SN observations directly constrains photon number violation. Any such systematic violations can then be interpreted 
as an opacity effect in the observed luminosity distance, parametrised through a generic opacity parameter, $\tau(z)$, as:

$$
d_{L, \mathrm{obs}}^{2}=d_{L, \text { true }}^{2} e^{\tau(z)} .
$$

Note that our "opacity" can have in principle both signs. In other words, this parametrisation also allows for apparent brightening of light sources, as would be the case, for example, if exotic particles were also emitted from the source and converted into photons along the line of sight [9]. From eq. (2.2) it is clear that the inferred distance moduli for the observed SNe picks an extra term which is linear in $\tau(z)$ :

$$
D M_{\text {obs }}(z)=D M_{\text {true }}(z)+2.5[\log e] \tau(z) .
$$

On the other hand, one can also use other determinations of distance measures, which are independent of $\tau$, to constrain possible deviations from eq. (2.1). This approach was initiated in reference [14] (see also [11, 12, 20, 21] for related earlier work) where the authors used measurements [22] of the baryon acoustic oscillation (BAO) scale at two redshifts, namely $z=0.20$ and $z=0.35$, to obtain a parameterization-independent upper-bound for the difference in opacity between these two redshifts, $\Delta \tau<0.13$ at $95 \%$ confidence. In reference [15] this constraint was improved (and also extended over a wider redshift range, but for a general parameterised form for $\tau$ ) by using, instead of measurements of the BAO scale at these two redshifts, measurements of cosmic expansion $H(z)$ from differential aging of LRGs at redshifts $z \lesssim 2$. This method of distance determination relies on the detailed shapes of galaxy spectra but not on galaxy luminosities, so it is independent of $\tau$.

In particular, the authors introduced a parameter $\epsilon$ to study deviations from the Etherington relation of the form:

$$
d_{L}(z)=d_{A}(z)(1+z)^{2+\epsilon},
$$

and constrained this parameter to be $\epsilon=-0.01_{-0.09}^{+0.08}$ at $95 \%$ confidence. Restricted to the redshift range $0.2<z<0.35$, where $\tau(z)=2 \epsilon z+\mathcal{O}\left(\epsilon z^{2}\right)$, this corresponds to $\Delta \tau<0.02$ at $95 \%$ confidence. In the following sections, we will apply similar constraints on different parametrisations of $\tau$ which correspond to particular models of exotic matter-photon coupling, namely axion-like particles (ALPs), chameleons, and mini-charged particles (MCPs).

Before moving to these models, we briefly update the above constraint on $\epsilon$ using the latest $H(z)$ data [23], which include two extra data points at redshifts $z=0.48$ and $z=0.9$, as well as the latest determination of $H_{0}$ [24]. Even though the addition of these two extra data points alone significantly improves the constraints of reference [15], the effect of $H_{0}$ is also quite significant, because it acts as an overall scale in the distance measures, which is marginalised over a Gaussian prior, and the measurement error in this determination is about half of that of the HST Key Project determination [25] used in [15].

Figure 1 shows the updated constraints obtained using the above data in combination with the SCP Union 2008 Compilation [16] of type Ia Supernova data, ${ }^{1}$ compared to the previous constraints of reference [15]. On the left, the darker blue contours correspond to the (two-parameter) $68 \%$ and $95 \%$ joint confidence levels obtained from SN data alone, while lighter blue contours are the corresponding confidence levels for $H(z)$ data. Solidline transparent contours are for joint $\mathrm{SN}+H(z)$ data. For comparison we also show the

\footnotetext{
${ }^{1}$ Note that we decide to use the SCP Union 2008 supernova sample rather than the more recent SDSS-II sample [26], because the Union sample extends to higher redshift and is thus best suited to be combined with the $H(z)$ data. A recent study using the SDSS-II sample, which also studies the distance duality relation considered here, can be found in ref. [27].
} 

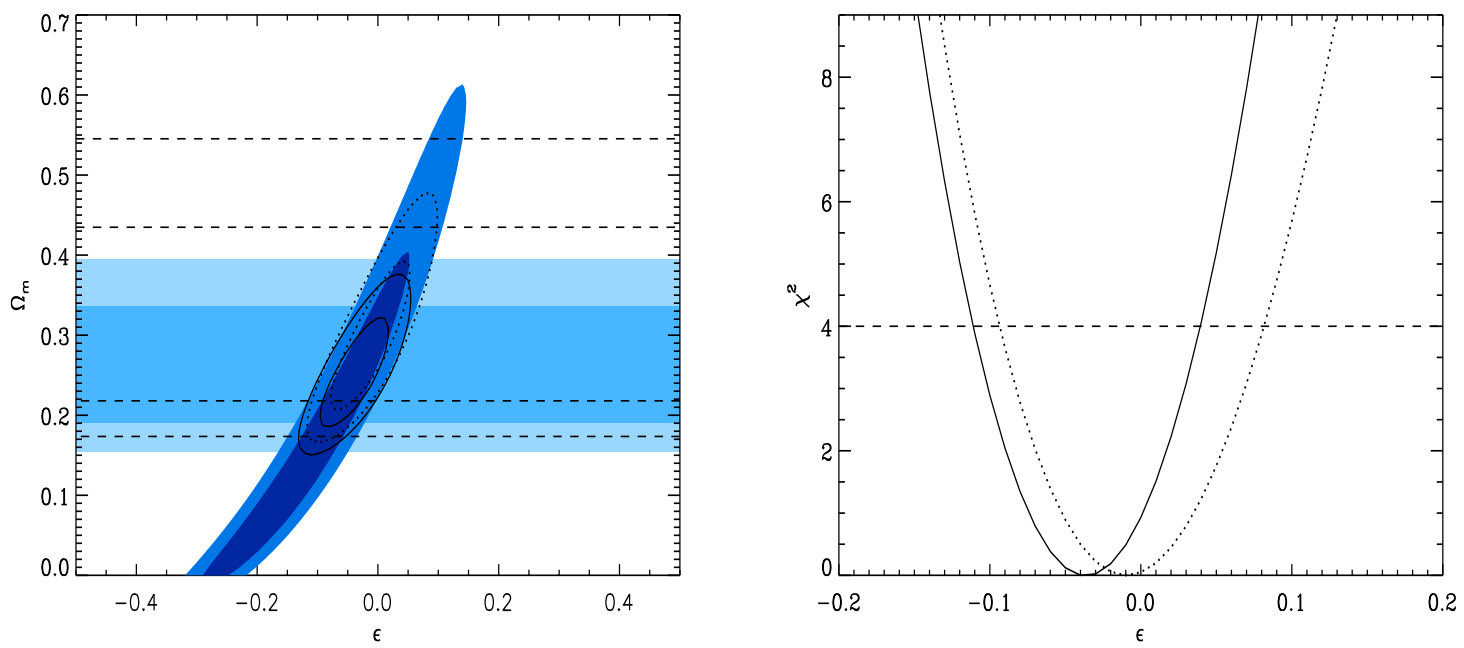

Figure 1. Updated constraints of reference [15], using the latest $H(z)$ data [23] and the Riess et al. determination of $H_{0}$ [24] in combination with the SCP Union 2008 SN Ia compilation. Left: Two-parameter constraints on the $\epsilon-\Omega_{m}$ plane. Darker blue contours correspond to $68 \%$ and $95 \%$ confidence levels obtained from SN data alone, lighter blue contours are for $H(z)$ data, and solid line transparent contours are for joint $\mathrm{SN}+H(z)$. Previous $H(z)$ and joint $\mathrm{SN}+H(z)$ from [15] are shown in dashed and dotted lines respectively. Right: One-parameter joint constraints on $\epsilon$ for the current analysis (solid line) and that of reference [15] (dotted line). The dashed line shows the 95\% confidence level, $\Delta \chi^{2}=2$.

previous $H(z)$ and $\mathrm{SN}+H(z)$ contours in dotted and dashed lines respectively. On the right we show one-parameter (marginalized over all other parameters) constraints on $\epsilon$, again for the current analysis (solid line) and for that of reference [15] (dotted). For the reader familiar with Bayesian methods, this plot corresponds to the posterior

$$
P(\epsilon \mid \mathrm{S}, \mathrm{E})=\int_{\Omega_{m}} \int_{H_{0}} P\left(\Omega_{m}, H_{0} \mid \mathrm{E}\right) P\left(\epsilon, \Omega_{m}, H_{0} \mid \mathrm{S}\right) \mathrm{d} \Omega_{m} \mathrm{~d} H_{0}
$$

where $P\left(\Omega_{m}, H_{0} \mid \mathrm{E}\right)$ and $P\left(\epsilon, \Omega_{m}, H_{0} \mid \mathrm{S}\right)$ are the posterior probabilities for the corresponding model parameters, given the $H(z)$ (Expansion) and SN (Supernovae) data respectively. These are given by the likelihoods of the two data sets in the model parameters, assuming Gaussian errors and using flat priors on all three parameters. In particular, we have taken $\epsilon \in[-0.5,0.5], \Omega_{m} \in[0,1]$ and $H_{0} \in[74.2-3 \times 3.6,74.2+3 \times 3.6]$ (Riess et. al. [24]), all spaced uniformly over the relevant intervals, in a flat $\Lambda$ CDM model. Similarly, the solid line transparent contours on the left plot of figure 1 correspond to taking only the integral over $H_{0}$ in the right hand side of eq. (2.5), yielding, therefore, the two-parameter posterior $P\left(\epsilon, \Omega_{m} \mid \mathrm{S}, \mathrm{E}\right)$.

As seen in figure 1 , the improvement in these constraints is significant. The new result on $\epsilon$, marginalised over all other parameters, is $\epsilon=-0.04_{-0.07}^{+0.08}$ at $95 \%$ confidence, which for redshifts between 0.2 and 0.35 (currently probed by BAO data), corresponds to a transparency (i.e., $\tau \geq 0$ ) bound $\Delta \tau<0.012$, a factor of two tighter than the result in reference [15]. ${ }^{2}$ We

\footnotetext{
${ }^{2}$ Note that the data slightly favour negative $\epsilon$ (thus the much stronger constraint on a positive $\Delta \tau$ ), but only at $\lesssim 1-\sigma$ level.
} 
now move on to study more general parametrisations of cosmic opacity, tailored for particular models of exotic matter coupled to photons.

\section{Axion-like particles and chameleons}

New scalar or pseudo scalar particles from physics beyond the standard model, here denoted as $\phi$, may couple to photons through

$$
\mathcal{L}_{\text {scalar }}=\frac{1}{4 M} F_{\mu \nu} F^{\mu \nu} \phi
$$

and

$$
\mathcal{L}_{\text {pseudo-scalar }}=\frac{1}{8 M} \epsilon_{\mu \nu \lambda \rho} F^{\mu \nu} F^{\lambda \rho} \phi
$$

where $M$ is the energy scale of the coupling (another widely used notation is $\left.g_{\phi \gamma}=1 / M\right), F_{\mu \nu}$ the electromagnetic field strength and $\epsilon_{\mu \nu \lambda \rho}$ the Levi-Civita symbol in four dimensions. Such fields are collectively known as Axion-Like Particles (ALPs), as a coupling of the form (3.2) arises for the axion introduced by Peccei and Quinn (PQ) to solve the strong CP problem [28]. Interestingly, these fields also arise naturally in string theory (for a review see [29]).

Axions, or axion-like-particles, can arise from field theoretic extensions of the standard model as Goldstone bosons when a global shift symmetry, present in the high energy sector, is spontaneously broken. In the PQ axion case, this symmetry is colour anomalous and the explicit breaking makes the axion pick up a small mass. This mass is, up to a modelindependent constant, proportional to the coupling (3.2). For a generic ALP, however, the mass is in principle independent of the strength of its coupling, and in particular can be zero if the related shift symmetry remains intact. That is, for instance, the case of Arions [30], the orthogonal combination of the PQ axion, if there are two independent colour anomalous shift symmetries.

Chameleon scalar fields are another very interesting type of ALPs [31]. They were originally invoked in $[32,33]$ to explain the current accelerated expansion of the Universe with a quintessence field which can couple to matter without giving rise to large fifth forces or unacceptable violations of the weak equivalence principle. The chameleon achieves this because its mass depends on the local energy density. The environmental dependence of the mass of the chameleon means that it avoids many of the constraints on the strength of the coupling, which normally apply to standard scalar and pseudo-scalar fields as they are derived from physics in dense environments. For a more detailed discussion see [34]. The cosmology of the chameleon was explored in detail in [35], the possibility of the chameleon coupling to photons was first discussed in [36] and such a coupling was shown to be generic in [31].

The Lagrangian terms given above mean that ALPs can affect the propagation of photons; in particular, if photons traverse a magnetic field there is a non-zero probability that they will oscillate into ALPs [37]. Notice however that only photons polarized perpendicular (parallel) to the magnetic field mix with scalar (pseudo-scalar) particles. Therefore, the interactions between photons and ALPs in the presence of a magnetic field not only imply that photon number is not conserved, but can also alter the polarization of the light beam. Both effects have been exploited in many searches for ALPs both in the laboratory and in astronomical observations, see [5] for a recent review. 


\subsection{Modelling the effects of ALPs}

The presence of ALPs will have an impact on observations of SNe if they are observed through intergalactic magnetic fields. In particular, it will lead to changes in the observed SN luminosities, in a redshift-dependent way. Many different mechanisms have been proposed which give rise to intergalactic magnetic fields, however we do not yet have convincing evidence from observations that they exist. A magnetic field coherent over the whole Hubble volume is limited, by observations of the CMB and Faraday rotation, to $B \lesssim 10^{-9} \mathrm{G}[38,39]$. Fields with shorter coherence lengths are also constrained. In particular, fields coherent on scales $\sim 50 \mathrm{Mpc}$ must satisfy $B \lesssim 6 \times 10^{-9} \mathrm{G}$, while fields coherent on scales $\sim$ Mpc must satisfy $B \lesssim 10^{-8} \mathrm{G}$ [39]. To explain the origin of galactic magnetic fields it is expected that intergalactic magnetic fields with coherence lengths $\sim$ Mpc are needed [40].

In a constant, coherent magnetic field the probability of a suitably polarized photon converting into an ALP after traveling a distance $L$ is given by [37]:

$$
P_{\gamma \rightarrow \phi}(L)=\sin ^{2}(2 \theta) \sin ^{2}\left(\frac{\Delta(L)}{\cos 2 \theta}\right)
$$

where

$$
\begin{aligned}
\Delta(L) & =\frac{m_{\mathrm{eff}}^{2} L}{4 \omega} \\
\tan 2 \theta & =\frac{2 B \omega}{M m_{\mathrm{eff}}^{2}},
\end{aligned}
$$

where $\omega=2 \pi \nu$ is the photon energy, $B$ is the strength of the magnetic field and $m_{\text {eff }}^{2}=$ $\left|m_{\phi}^{2}-\omega_{P}^{2}\right|$, with $m_{\phi}$ the mass of the ALP and $\omega_{P}^{2}=4 \pi^{2} \alpha n_{e} / m_{e}$ the plasma frequency of the medium which acts as an effective mass for the photons ( $\alpha$ is the fine structure constant, $n_{e}$ the local number density of electrons, and $m_{e}$ the mass of the electron). In what follows we restrict our attention to very light fields, $m_{\phi}^{2}<\omega_{P}^{2}$, where observations of the opacity of the universe have the most power to constrain the strength of the coupling for the ALP to photons.

However, the intergalactic magnetic field is not coherent from the earth to the supernovae. We model its fluctuations using the cell magnetic field model, whereby we assume that the magnetic field is split up into a large number of equally sized domains. Within each domain the magnetic field is constant, its strength being the same in all domains, while its orientation is chosen randomly. The cell magnetic field model is the simplest choice we can make to approximate the structure of astrophysical magnetic fields, and is commonly used both in the study of ALPs and of astrophysical magnetic fields. A more accurate choice for a model of the magnetic field would be to assume a power spectrum of its fluctuations. However, at high frequencies, $\Delta \ll \pi / 2$, the cell and power spectrum models give the same results, and at lower frequencies the cell model captures all the qualitative features of ALPphoton interactions, but underestimates the probability of conversion [41, 42]. Therefore, using the cell magnetic field model will give rise to conservative constraints.

Clearly, we also need to know the plasma frequency of the intergalactic medium along the line of sight. This is quite a complicated issue because no measurements of the electron density are available in the large voids of the interstellar medium. A large-scale average can be easily inferred from the total amount of electrons determined by CMB estimation of the baryon to photon ratio giving $\omega_{P} \simeq 1.8 \times 10^{-14} \mathrm{eV}$ today, see e.g. [43]. Note, however, that average values up to a factor of 15 smaller were considered plausible in [44]. Since there is no 
easy way out of this conundrum, the accepted approach is to assume that $\omega_{P}^{2}$ is homogeneous and equal to the average value. To check the dependence of the results on this assumption, we will finally allow a range of a couple of orders of magnitude around the average.

As we are interested in the transparency of the Universe out to redshifts $z \sim \mathcal{O}(1)$ we must also take into account the redshift evolution of the environment that causes mixing between photons and scalars. Assuming the magnetic fields are frozen into the plasma, their strength scales as $B(z)=B_{0}(1+z)^{2}[40]$ while $\omega(z)=\omega_{0}(1+z)$ and $\omega_{P}^{2}(z)=\omega_{P 0}^{2}(1+z)^{3}$ (since it is proportional to the electron density). Here, the subscript 0 indicates values in the present epoch. The physical length of a magnetic domain scales as $L(z)=L_{0}(1+z)^{-1}$ as long as it is smaller than the Hubble radius. Then the two parameters that appear in the probability of conversion (3.3), $\theta$ and $\Delta$, redshift as

$$
\begin{aligned}
\Delta(z) & =\Delta_{0}(1+z), \\
\tan 2 \theta(z) & =\tan 2 \theta_{0} .
\end{aligned}
$$

There are two limits in which the expression for the conversion probability in one domain simplifies notably, the coherent and incoherent regimes. In the coherent regime, the argument of the sinus in eq. (3.3) is smaller than 1 and, taking $\sin x \sim x$, the probability takes a very simple expression:

$$
P_{\gamma \rightarrow \phi}(z) \approx\left(\frac{B_{0} L_{0}}{2 M}\right)^{2}(1+z)^{2} \quad(\text { coherent }) .
$$

Note that, even if the probability is frequency-dependent in a generic case, in the coherent limit this dependence vanishes.

On the other hand, if the argument of the sinus - which is energy dependent - is very large, then a large number of oscillations will happen in a finite energy bin, which would average out the sinus to $1 / 2$. In this case we find

$$
P_{\gamma \rightarrow \phi}(z) \approx \frac{1}{2}\left(\frac{2 B_{0} \omega_{0}}{M \omega_{P, 0}^{2}}\right)^{2} \quad \text { (incoherent) } .
$$

These approximations are only valid for small values of $P_{\gamma \rightarrow \phi}$. Finally, note that from now on we will drop the subscript 0 for today's values of the various parameters and make the redshift dependence explicit.

In the above limits, the redshift dependence is very simple and a system of axionlike particles and photons can be evolved analytically through a large number of randomly oriented magnetic domains. Let us introduce the notation $P$ for the transition probability $P_{\gamma \rightarrow \phi}$ in one domain today. Assuming that the magnetic fields and intergalactic medium do not evolve with redshift, the photon survival probability was first computed in [46]. This is then exactly valid in the incoherent regime of eq. (3.9). The finite probability of conversion gives rise to an apparent change in luminosity distance. In particular, if photons are converted to ALPs along the line of sight, then the inferred and true and luminosity distance squared (cf eq. (2.2)) will differ by a factor $\mathcal{P}(z)$, which in this case reads:

$$
\mathcal{P}(z)=A+(1-A) \exp \left(-\frac{3}{2} \frac{y(z)}{L} P\right),
$$

where $y(z)$ is the comoving distance to the source. Physically, $\mathcal{P}(z)$ is the average probability that a photon emitted by a supernova at redshift $\mathrm{z}$ is observed by us after traversing the 
magnetic fields in the intergalactic medium. The above formula is valid for small $P$; in the case where $P$ is of order unity, one should replace $3 P / 2 \rightarrow-\ln (1-3 P / 2)$. We have allowed for an initial flux of axions $I_{\phi}\left(z_{I}\right)$ and defined ${ }^{3}$

$$
\mathcal{P}(z)=\frac{I_{\gamma}(0)}{I_{\gamma}\left(z_{I}\right)} \quad ; \quad A=\frac{2}{3}\left(1+\frac{I_{\phi}\left(z_{I}\right)}{I_{\gamma}\left(z_{I}\right)}\right) .
$$

In the coherent regime, we wish to allow for additional effects due to the evolution of the magnetic fields with redshift. The fluxes of scalars and photons at the end of the $n$-th domain are related to the fluxes at the beginning of the domain by

$$
\left(\begin{array}{l}
I_{\gamma}(z) \\
I_{\phi}(z)
\end{array}\right)_{(n+1)}=\left(\begin{array}{cc}
1-\frac{1}{2} P(1+z)^{2} & P(1+z)^{2} \\
\frac{1}{2} P(1+z)^{2} & 1-P(1+z)^{2}
\end{array}\right)\left(\begin{array}{c}
I_{\gamma}(z) \\
I_{\phi}(z)
\end{array}\right)_{(n)}
$$

and we want to multiply a large number of these matrices together. Usefully, the matrix is diagonalisable by a redshift independent transformation, so that, after passing through $N$ domains, the photon survival probability can be easily shown to be

$$
\mathcal{P}(z)=A+(1-A) \prod_{j=1}^{N}\left(1-\frac{3}{2} P\left(1+z_{j}\right)^{2}\right),
$$

where $\left\{z_{j}\right\}$ is a collection of redshifts in the range $(0, z)$, equally spaced in comoving length. If the number of domains is large, we can approximate the product by an integral to get

$$
\mathcal{P}(z)=A+(1-A) \exp \left(-\frac{P}{H_{0} L} \frac{H(z)-H_{0}}{\Omega_{m} H_{0}}\right) .
$$

This resembles the expression for the photon survival probability when the evolution of the background is neglected, equation (3.10), but has a stronger $z$-dependence at large redshifts. Both formulae give the same results in the small $z$ regime, since $y(z) \simeq z / H_{0}$ and $\left(H / H_{0}-\right.$ 1) $/ \Omega_{m} \simeq 3 z / 2$.

The redshift evolution of eqs. (3.10) and (3.15) is absent when $A=1$. When this is the case, the initial flux of photons and scalars is already thermalised $I_{\gamma}\left(z_{I}\right)=2 I_{\phi}\left(z_{I}\right)$ so that,

\footnotetext{
${ }^{3}$ Very recently it was pointed out that this formula is actually an averaged formula over different realisations of the configuration of magnetic field domains along a line of sight [47, 48]. As such, it is in principle not valid for a single source, whose light only travels through a concrete realisation of the magnetic field domain structure. The authors of [47] calculated an analytical estimate for the dispersion around the mean — although the distribution is non-Gaussian and in general asymmetrical so a meaningful confidence interval has to be computed numerically — for $A=2 / 3$. Using $x=P y(z) / L$, the result reads

$$
\delta \mathcal{P}=\sqrt{R-\mathcal{P}^{2}} \quad \text { with } \quad R=\frac{49+50 \exp (-3 x / 2)+6 \exp (-5 x)}{105},
$$

which falls in the range $\sim(0,1 /(3 \sqrt{5}))$, i.e. the dispersion is below $15 \%$. In the Hubble diagram, this corresponds to a maximum dispersion of 0.2 magnitudes, which is the typical dispersion in the SN data we use. This otherwise suggestive fact, implies that confronting the observed with the predicted dispersion can only potentially constrain a regime of large values of $x$ - what we will later introduce as strong mixing regime - and most likely with small significance. We conclude that it is more promising to confront the mean value, given by eq. (3.10), with observations. In fact, as we shall see below, when constraining "opacity" as a function of redshift, for every reasonable redshift interval we may consider, there will be several data points corresponding to different supernovae at different positions in the sky, yielding an effective average; therefore in the following we can obviate the implications of the uncertainty in the value of $\delta P$.
} 
on average, the effect of photons converting into scalars is compensated by that of scalars converting into photons, and thus no net effect of the mixing is seen.

In summary, the luminosity distance to a supernova is modified by an overall, redshiftdependent factor:

$$
d_{L} \rightarrow d_{L} / \sqrt{\mathcal{P}(z)} .
$$

As mentioned in section 2, the effect of ALP-photon mixing described above can be interpreted as "opacity", generally of both signs, so that

$$
\tau(z)=-\ln \mathcal{P}(z) .
$$

Note finally that $A$ and $P$ and therefore also $\mathcal{P}$ and $\tau$ might in principle depend on the frequency of light. In 3.4 and 3.5 we will comment further on the impact that such a dependence might have. We now proceed to constrain the possibility of mixing between scalars and photons, through their effects on cosmic opacity. Again, there are two regimes in which analytical insight can be reasonably expected.

\subsection{Weak mixing}

We begin by considering the case where the sum of the $\gamma \rightarrow \phi$ conversion probabilities in all the domains is smaller than unity $\left(N P_{\gamma \rightarrow \phi} \ll 1\right)$. In this limit the effects of ALP-photon mixing are always small so it is known as weak mixing regime. When the redshift evolution of the background is neglected, the probability of photon survival can be found [34, 41, 42] to be

$$
\mathcal{P}(z)=1-(1-A) \frac{3}{2}\left(\frac{y(z)}{L}\right) P .
$$

In the low redshift regime, both the redshift dependent and independent equations give the same opacity

$$
\tau(z)=(1-A) \frac{3}{2} \frac{P}{H_{0} L} z .
$$

Note that this is exactly of the form used in section $2, \tau(z)=2 \epsilon z$. We can already obtain a first estimate of our bounds by using our improved constraint $\epsilon<0.04$ at $95 \%$ confidence. Using $H_{0} \simeq 74.2 \mathrm{~km} / \mathrm{s} \mathrm{Mpc}^{-1}$ we obtain

$$
P \lesssim 4 \times 10^{-5} \frac{L}{\mathrm{Mpc}}\left(\frac{1 / 3}{1-A}\right) .
$$

Note that $A \simeq 1$ cannot be constrained. As mentioned before, this situation corresponds to the initial flux of axions and photons having almost thermalized abundances (see eq. (3.12)). A thermalized, or nearly thermalized, axion/photon flux has very small redshift dependence (since the mixing tends to thermalization, but this is almost complete before leaving the SN source) and thus distance measures have no constraining power.

Two further comments are in order. Our bound of eq. (3.20) corresponds to the argument of the exponential taking values around $-0.081 z /(1-A)$. This corresponds to the start of the exponential regime for the larger redshifts, so it is consistent with the Taylor expansion adopted, unless $A$ is fine-tuned to 1. Finally, note that the bound on $\epsilon$ has been obtained considering redshifts up to $z \sim 1.5$, while the validity of eq. (3.19) is only ensured for small redshifts. For this reason this bound should be considered as an order-of-magnitude estimation; we present an accurate, numerical study, in section 3.4. 


\subsection{Strong mixing}

The other analytically analysable limit of eqs. (3.10) and (3.15) is when the sum of the conversion probabilities in all domains is very large, $N P \gg 1$. If the mixing between axionlike particles and photons is strong, then, on average, one-third of any initial flux will be converted into axion-like particles and two thirds into photons. This can cause substantial changes to the apparent supernovae luminosities. It was initially speculated [6] that this could account for the dimming of SNe without the existence of dark energy, however this has now been excluded by observations of the CMB [49]. If there is a large initial flux of axion-like particles from SNe, then the SN images can be brightened by strong mixing between ALPs and photons in the intergalactic medium [9].

In the strong mixing limit both equations (3.10) and (3.15) give the same result, $\mathcal{P} \sim A$, that is, a constant independent of redshift. It is clear that our methods cannot constrain such a possibility, which would be equivalent to a change of the normalization of the SN luminosity. Imposing $N P>1$ at the closest $\mathrm{SN}$ redshifts up to $z \sim 0.01$, we find

$$
P>0.015 \frac{L}{\mathrm{Mpc}} .
$$

We cannot constrain this range of probabilities as the mixing is too strong and the redshift dependence of the opacity is washed out.

\subsection{Numerical results}

In this subsection we turn our estimates into real constraints by means of a full likelihood analysis. Note that our results only depend on the conversion probability per comoving length $P / L$, so we cannot constrain $P$ and $L$ independently.

As we have already pointed out, both the photon transmission probability and the ALP flux parameter $A$ can in principle depend on the frequency of the SN photons. However, it is experimentally known that SN dimming is frequency independent to a precision of $\sim 3 \%[44,45]$. This observation has been used to provide constraints on photon-ALP mixing from SN observations, to which we shall come back later. This frequency dependence could in principle affect also the determination of $H(z)$ we are using. However, the bulk of the signal in the differential aging method comes from narrow-band features in the galaxy spectra. In particular, the most prominent spectral feature relevant for the aging method is the $4000 \AA$ break, which is about $100 \AA$ wide. Since we are focusing in constraining the universe opacity by testing the Etherington relation and not by those spectral features, we assume for the moment that the parameters $P$ and $A$ are frequency independent. As we shall argue later when we interpret our results in terms of particle physics models this procedure is either justified or produces conservative bounds.

In figure 2 we show our results marginalized over $\Omega_{m}$ and $H_{0}$. The dark and light contours represent $68 \%$ and $95 \%$ joint confidence levels respectively, using the SN data only (left) and joint $\mathrm{SN}+H(z)$ data (right). In the upper panels, we have used eq. (3.15), thus taking into account the redshift dependence of the background, while in the lower panels we used eq. (3.10), ignoring these effects.

The strong and weak mixing limits described in sections 3.3 and 3.2 are clearly visible in figure 2. We represent them schematically by the green and red lines below and above the boundaries of our plots respectively. For very small conversion probability $P \lesssim 10^{-5}$ we are in the weak mixing limit and our constraints become weak because of the lack of photon-ALP 

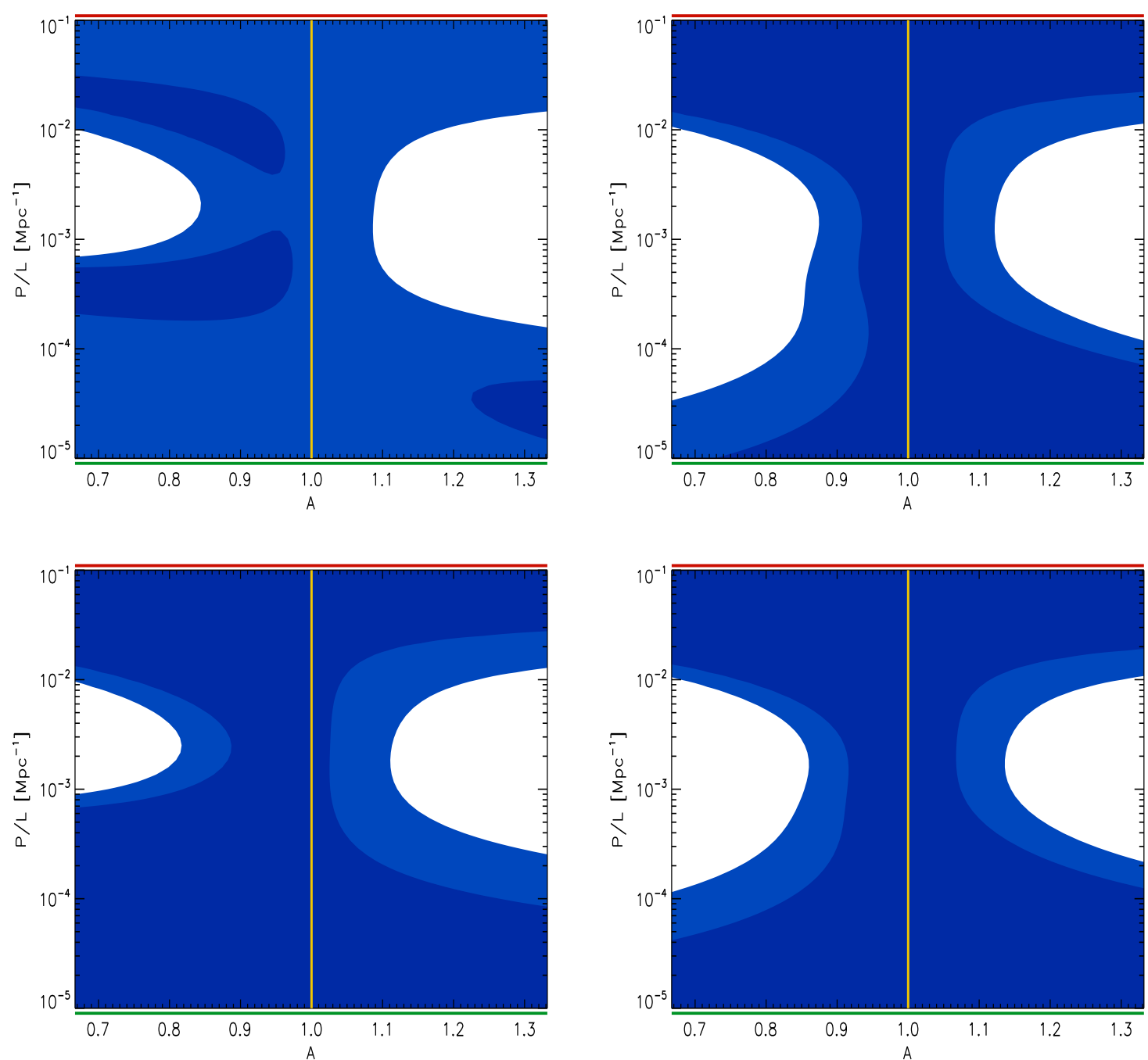

Figure 2. Two parameter constraints in the $A-P / L$ plane for general ALPs considering redshift dependence of the background (upper panels) and neglecting it (lower panels). Contours represent the $68 \%$ (dark) and 95\% (light) confidence levels. For the left panels we have used SN data only, while in the right panels we show the joint $\mathrm{SN}+H(z)$ analysis. The contours are marginalized over cosmologies and $H_{0}$. While redshift dependence introduces $\mathcal{O}(1)$ effects, which change the structure of the $68 \%$ CL contours, the $95 \%$ CL contours are very similar in the two cases.

mixing. On the other extreme, when the probability is very strong $\left(P \gtrsim\right.$ few $\left.\times 10^{-2}\right)$ the photons and axions mix until thermalization and the redshift dependence of the opacity is lost, so, again, our constraints become weak. Finally we can also observe that a band around $A=1$ (yellow line) again cannot be constrained. $A=1$ means an equilibrated photon-ALP flux from the SNe such that photon $\rightarrow \mathrm{ALP}$ conversions are compensated with the reverse process, making the photon number constant, i.e. redshift-independent.

The first notable feature is that, as expected from our estimations in the first section, our $2-\sigma$ bounds (white regions) are significantly improved when including the $H(z)$ data (right panels) compared to using SN data alone (left panels). The improvement is particularly 
visible in the weak mixing regime. This will show more clearly in the constraints on the $P / L-\Omega_{m}$ plane, which we will present below. We shall comment on this improvement separately in each case of interest.

The second effect is the importance of including the redshift dependence of the magnetic fields, the photon frequency and the physical length of domains, that is, the difference between using eqs. (3.10) and (3.15). The 95\% C.L. constraints are improved only slightly by including the redshift dependence (upper panels), compared to a background that does not evolve (lower panels), the exception being the factor of 3 improvement at $A \simeq 2 / 3$ in the $\mathrm{SN}+H(z)$ analysis (right panels).

A peculiar feature appears in the upper left plot, corresponding to a redshift-dependent background and using SN data only. There, the $68 \%$ C.L. contour shows not one, but three regions where photon-ALP mixing improves the fitting of the data compared to a standard $\Lambda \mathrm{CDM}$ cosmology. The lower-left region corresponds to the parameters invoked by Csaki et al. [6] to explain SN dimming without cosmic acceleration. It is remarkable that the data show this preference much more sharply in the redshift-dependent background case given by eq. (3.15) than in the simpler redshift-independent case used in [6] (eq. (3.10)). As we show in the next section, our numerical analysis implies that this island in parameter space corresponds to $\Omega_{m}<1$, which, in the absence of a cosmological constant, implies a non-flat geometry. Most importantly, the joint $\mathrm{SN}+H(z)$ analysis (upper right panel) rules out this region.

The preferred region at large $A$ and small $P / L$ also deserves some comments. A value of $A$ greater than unity produces an increase of the SN luminosity with redshift because the $\mathrm{SNe}$ would shine more ALPs than photons. In figure 1 we have shown that, because of the pronounced degeneracy, SN data alone show a slight preference for this scenario since the $68 \%$ C.L. contour is almost completely located at negative values of our opacity parameter $\epsilon$. In the joint $S N+H(z)$ analysis, the trend is softened but, still, small negative values (slight SN brightening) are slightly preferred, even though this is not statistically significant. Therefore, in the remaining panels of figure 2 this possibility cannot be excluded, but the statistical preference for this region decreases compared to the rest of the allowed parameter space. The fact that photon-ALP mixing alleviates the tension between SN data and standard rulers was already pointed out in [9]. The value of $P / L \sim 2 \times 10^{-5} \mathrm{Mpc}^{-1}$ implies that the required effect on the opacity is small. In the weak mixing limit, the opacity is proportional to $(1-A) P / L$ (see eq. (3.19)), making $A$ and $P / L$ correlated. This region is therefore expected to extend further right and down. Notably, these small values of $P / L$ are not excluded by other arguments (see next section), although it seems difficult to conceive a model where $A$ is sufficiently large.

\subsection{Constraints}

We can now interpret our results in terms of constraints on the physical parameters of our Lagrangian. There are several cases to consider.

\subsubsection{Axion-like-particles}

If ALPs have no other interactions than the two-photon coupling, then they were shown to contribute very little to the SN luminosity [46], corresponding to the case $A \simeq 2 / 3$. It is evident from figure 2 that only a range of conversion probabilities around $P / L \sim \mathcal{O}\left(10^{-3}\right)$ can be excluded. Figure 3 shows 1- and 2- $\sigma$, two-parameter likelihood contours on the $P / L-\Omega_{m}$ plane after marginalization over $H_{0}$. Note that even the SN constraints alone (dark blue contours) rule out this model as an alternative to a cosmological constant, at greater than 

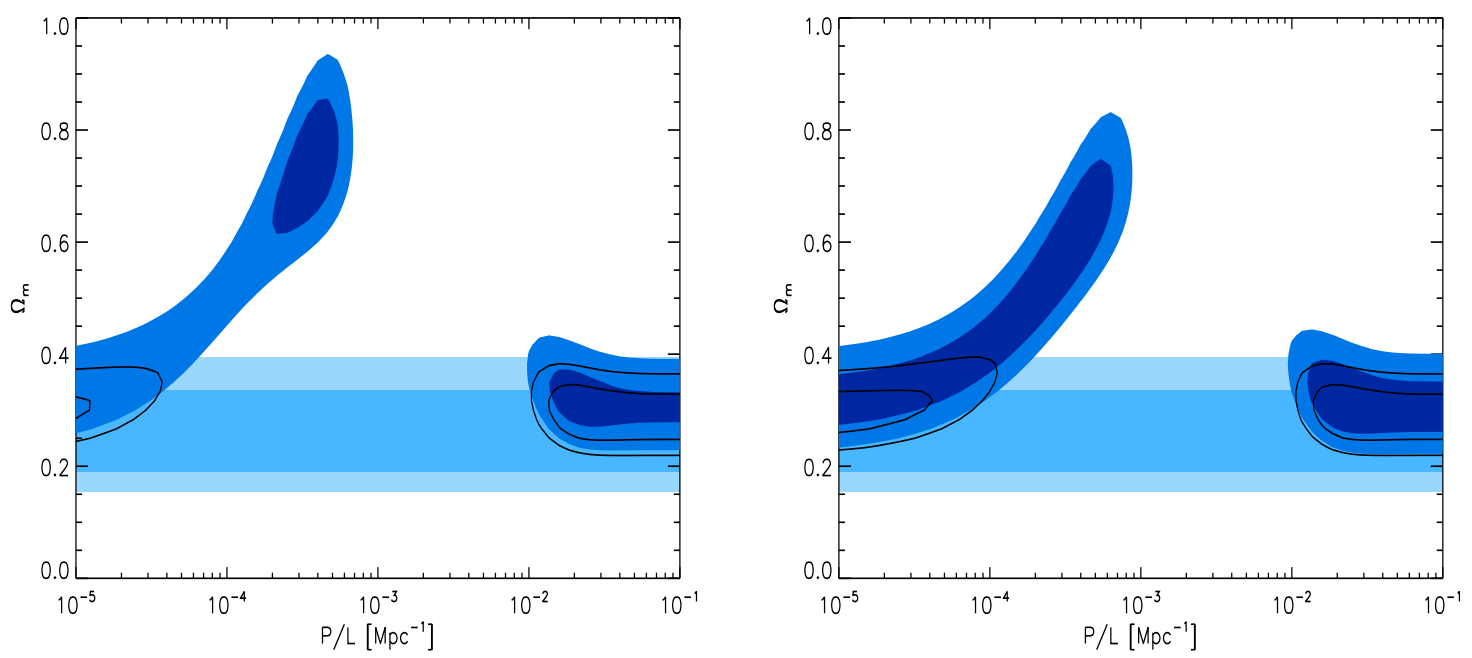

Figure 3. Confidence levels $(68 \%$ and $95 \%)$ on the $P / L-\Omega_{m}$ plane for the simplest axion-likeparticle model $(A=2 / 3)$. The small and large $P$ regions correspond to the weak and strong mixing regimes respectively. Dark blue contours show constraints from SN data only, light blue from $H(z)$ data, and solid line contours from joint $\mathrm{SN}+H(z)$. In the left panel, the redshift dependence of the background is taken into account, while in the right panel these affects are ignored.

3- $\sigma$ significance. However, there is still significant degeneracy in the weak mixing regime, and a value for $\Omega_{m}$ greater than 0.8 is still allowed at the $2-\sigma$ level, this constraint being slightly weaker when the background redshift dependence is taken into account (left). Including the $H(z)$ data (light blue contours) breaks this degeneracy, yielding strong joint constraints in $P / L-\Omega_{m}$ (solid line contours).

We can now translate the bounds on $P / L$ into bounds on the strength of the ALP coupling to photons. Since the coupling always appears multiplied by the magnetic field (which is also unknown) we find convenient to quote bounds on the combination $B / M$. Let us also define appropriately normalised values of the magnetic field strength $B$ and the energy-scale of the the axion-photon coupling $M$, as

$$
B_{\mathrm{nG}}=\frac{B}{1 \mathrm{nG}} \quad ; \quad M_{10}=\frac{M}{10^{10} \mathrm{GeV}} .
$$

In figure 4 we show our constraints for the case $L=1 \mathrm{Mpc}$ as a function of the uncertain value of the average electron density, or, equivalently, the plasma frequency. To get rid of the oscillations of eq. (3.3), which not only will be averaged out by energy binning but also by small fluctuations in the sizes of the domains and the values of the plasma frequencies, we propose the substitution $\sin ^{2} x \rightarrow\left(1-\exp \left(-2 x^{2}\right)\right) / 2$, which reproduces the coherent and incoherent limits. The exclusion limit is a horizontal band which bends upwards around $n_{e} \simeq 0.2 \times 10^{-7} \mathrm{~cm}^{-3}$. The horizontal part corresponds to the coherent case, where the $n_{e}$ dependence drops out of $P$, while the diagonal band corresponds to the incoherent case where $n_{e}$ suppresses $P$. Note that the average electron density today is $n_{e} \simeq 10^{-7} \mathrm{~cm}^{-3}$, near the transition between the two regimes.

Our bound on $P$ assumed a frequency independent dimming but this does not hold in the incoherent case, cf. eq. (3.9). Since the data are compatible with a frequency indepen- 


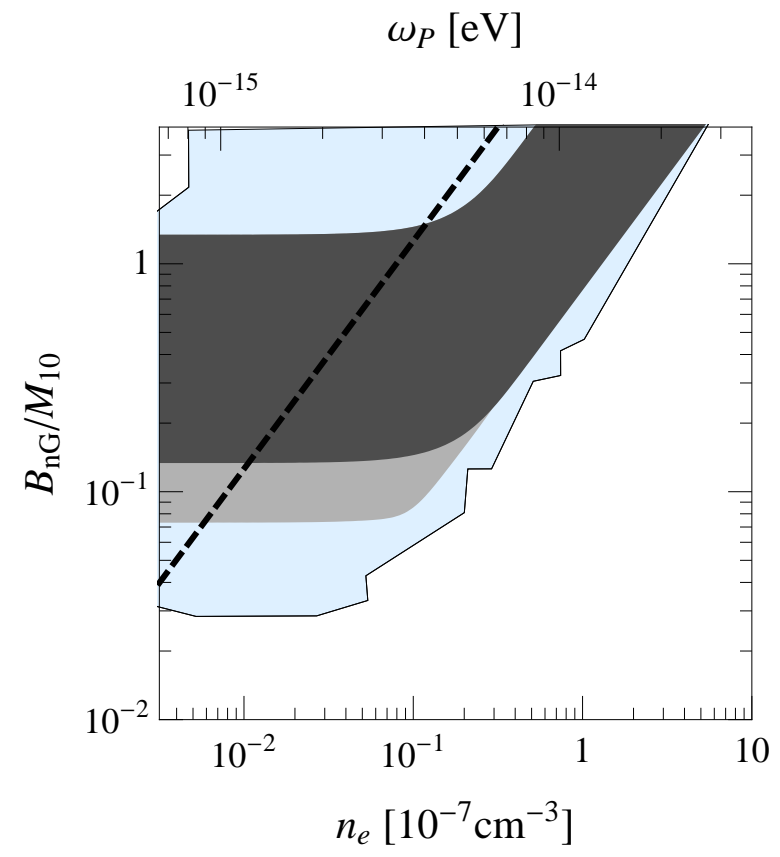

Figure 4. Bounds for the product of ALP coupling times magnetic field $B_{\mathrm{nG}} / M_{10}$ as a function of the average electron density or corresponding plasma frequency. The grey regions are excluded at 95\% C.L. for $L=1 \mathrm{Mpc}$ in the redshift-independent (dark) and dependent (light) cases. Also shown are the regions constrained from CMB [49] (above the dashed line), which dominate at low $n_{e}$, and QSO [50] (blue) spectral distortions.

dent dimming to a good accuracy any frequency dependence would worsen the $\chi^{2}$ making our bounds stronger. In turn this suggests that our procedure of ignoring this dependence produces conservative bounds. In summary, while in the coherent regime $\left(n_{e} \lesssim 10^{-7} \mathrm{~cm}^{-3}\right)$ our bounds are accurate because there is no frequency-dependent dimming, they become increasingly underestimated, i.e. conservative, as we increase $n_{e}$ and we enter more deeply into the incoherent regime.

In the same figure, we have also reproduced the constraints of Mirizzi et al [49] from distortions of the CMB (region above the dashed line) and those of [50] (blue region) from QSO spectra (see also footnote 2 in [49] and [51, 52]). For $n_{e} \gtrsim 10^{-9} \mathrm{~cm}^{-3}$ our bounds are stronger than the CMB ones while still competitive with the QSO bounds. Our approach provides a complementary, independent way to obtain these constraints. Each of the three approaches reported in the figure (especially the present constraint and the QSO one) is affected by different, unrelated, systematics: their agreement promotes one's confidence in these results.

\subsubsection{Chameleons}

Unlike the simple ALP case studied above, the chameleon Lagrangian contains non-linear selfinteractions of the scalar field in order that the mass of the scalar may become dependent on the density of its environment. This introduces the possibility of having $A \neq 2 / 3$.

There are three possible cases: i) either $A \simeq 2 / 3$ because few chameleons are produced in the SN, ii) $A-1 \ll 1$ because they interact so strongly in the SN that photons and 
chameleons thermalize their fluxes within the $\mathrm{SN}$, and iii) the intermediate case where a significant number of scalar particles are produced in the interior of the SN, but, yet, photonchamelon interactions are not strong enough to thermalise the chameleon population with that of the photons before they leave the SN.

The first case is morphologically equivalent to the previous ALP case so the conclusions of the last section hold. In the second case we see from figure 2 that we cannot constrain any value of the probability of conversion. Our sensitivity is at most $|A-1| \gtrsim 0.1$ but only in a very narrow range of $P$ around $10^{-3} \mathrm{~L} / \mathrm{Mpc}$. Therefore, we cannot exclude the possibility that photons and chameleons mix strongly in the intergalactic medium, even though, in order to realise this scenario fully, more work is required to understand the possible fluxes of chameleons from SNe. For the third case we can obtain a constraint on the $P / L-\Omega_{m}$ plane by marginalizing over $A$ in the physically reasonable range $[2 / 3,4 / 3]$ (refer to eq. (3.12)). Figure 5 shows our results in this case together with the corresponding constraint on the $A-\Omega_{m}$ plane after marginalising over $P / L$ in the range $\left[10^{-5}, 10^{-1}\right]$.

Note that, in agreement with our analysis of the simplest ALP case with $A=2 / 3$, our constraints exclude these models as an alternative to a cosmological constant at greater than $3-\sigma$ if the Universe is set to be spatially flat, while $\Omega_{m}>0.8$ is excluded at $2-\sigma$ even for SN data alone, and at much higher statistical significance when $H(z)$ data are included. This is true both when the redshift dependence of the background is included (upper panels) or neglected (lower panels), the constraint in the latter case being somewhat stronger.

\section{Mini-charged particles/hidden photons}

New particles with a small unquantized charge have been investigated in several extensions of the standard model $[53,54]$. In particular, they arise naturally in extensions of the standard model which contain at least one additional $\mathrm{U}(1)$ hidden sector gauge group [53, 55]. The gauge boson of this additional $\mathrm{U}(1)$ is known as a hidden photon, and hidden sector particles, charged under the hidden $\mathrm{U}(1)$, get an induced electric charge proportional to the small mixing angle between the kinetic terms of the two photons. In string theory, such hidden $\mathrm{U}(1) \mathrm{s}$ and the required kinetic mixing are a generic feature [56-60]. Hidden photons are not necessary however to explain mini-charged particles, and explicit brane-world scenarios have been constructed [54] where MCPs arise without the need for hidden photons.

The existence of low-mass MCPs can have a tremendous impact on photon propagation over cosmological distances. Photons from a given source can for instance pair produce MCPs with CMB photons $\gamma+\gamma_{\mathrm{CMB}} \rightarrow \psi^{+}+\psi^{-}$, leading to a new form of opacity. However, this process is generally more noticeable for CMB photons rather than those of higher energy, both because the CMB spectrum was measured by the FIRAS experiment to be a perfect blackbody with a typical accuracy of $10^{-4}$, and also because the cross-section is inversely proportional to the center-of-mass energy. The impact of the existence of MCPs for CMB distortions was studied in [61], where a limit for the minicharge $q_{\epsilon}<4 \times 10^{-8}$ (measured in units of the electron's charge) was derived for 4-component Dirac MCPs.

A more relevant source of opacity was pointed out in [10], following the work of [62, 63]. Photons propagating in a background magnetic field can actually pair-produce MCPs without the need for a second photon in the initial state. This is due to the fact that in a background field energy-momentum conservation is non-trivial. Indeed, the magnetic field acts as a refractive medium where both the mini-charged particles and photons acquire a non-trivial dispersion relation, i.e. effective masses. In the most interesting case, the effective photon 

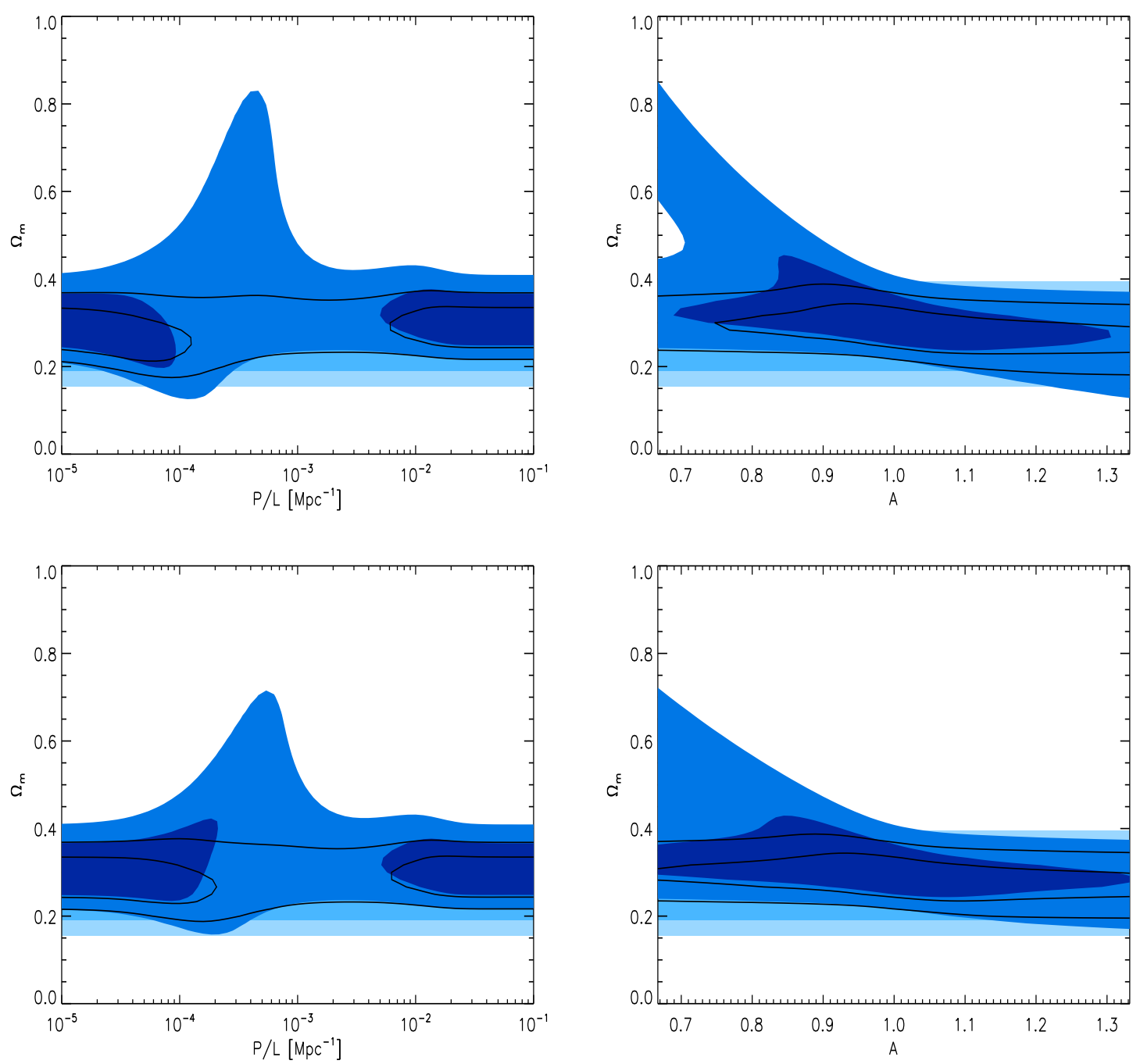

Figure 5. $68 \%$ and $95 \%$ confidence levels (two parameters) on the $P / L-\Omega_{m}$ (left) and $A-\Omega_{m}$ (right) planes, for chameleons with a prior $A \in[2 / 3,4 / 3]$. In the upper we have taken into account the order $\mathcal{O}(1)$ effects arising from the redshift dependance, while in the lowers panels we have ignored these effects. Dark blue contours are for SN data only, light blue ones for $H(z)$ data, and black transparent contours are for the joint $\mathrm{SN}+H(z)$ analysis.

mass is larger than that of a MCP pair, and the $\gamma \rightarrow \psi \bar{\psi}$ process happens at a rate

$$
\kappa=\frac{e^{8 / 3}}{4 \Gamma\left(\frac{1}{6}\right) \Gamma\left(\frac{13}{6}\right)}\left(\frac{2 B^{2} q_{\epsilon}^{8}}{3 \omega}\right)^{1 / 3} f,
$$

where $q_{\epsilon}$ is the MCP electric charge in units of the electron's charge $e$, and $f$ is an order one factor which depends on the nature of the MCP and the photon polarization with respect to the magnetic field, assumed again to be transverse to the photon direction of motion ( $f=1,2 / 3$ for parallel and perpendicular polarizations respectively if the MCP is a Dirac spinor, and $f=1 / 12,1 / 4$ if the MCP has spin- 0$)$. $\Gamma$ denotes the usual $\Gamma$-function. The above 
formula is valid in the deep non-perturbative regime, where the adiabatic condition

$$
\frac{3}{2} \frac{\omega}{m_{\psi}} \frac{e q_{\epsilon} B}{m_{\psi}^{3}} \ll 1
$$

holds (hence the unusual scaling with the charge, $\left(e q_{\epsilon}\right)^{8 / 3}$ ). Note that in this regime the process is independent of the MCP vacuum mass, $m_{\psi}$, but this parameter still enters through the adiabatic condition (4.2). For the value of $\kappa$ in the non-adiabatic regime we refer to appendix A of [63].

The MCP pair production process damps the photon flux according to the usual decay law, so the photon survival probability after traveling physical distance $L$ will be given by

$$
\mathcal{P}(L)=\exp \left(-\int_{0}^{L} \kappa(L) d L\right),
$$

where $L$ is redshift-dependent. Using the redshift dependencies quoted in the discussion around eq. (3.6), $\kappa$ redshifts $^{4}$ as $\kappa(1+z)$. This leads us to

$$
\mathcal{P}(z)=\exp (-\kappa y(z)),
$$

where $y(z)$ is the comoving distance to the source. Note that this expression can be recovered from the ALP case, eq. (3.9), in the $A \rightarrow 0$ limit and substituting $3 P /(2 L) \rightarrow \kappa$.

As was noted in $[10,64]$ the above expression does not hold in what is probably the most interesting case, in which the MCPs arise from kinetic mixing. In that situation, photon to hidden photon oscillations also have to be taken into account and, most surprisingly, they tend to suppress the photon disappearance! In this scenario both photons and hidden photons get an effective mass from the magnetic-field-dressed MCP. However, the coupling of hidden photons to the MCP particles is much stronger than the corresponding coupling for photons, so the refractive effect (the effective mass) is always larger. The large mass of the hidden photon acts to suppress the mixing angle between photons and MCPs, in a similar manner a large plasma frequency (effective mass for the photon) suppresses the mixing between photons and ALPs in the previous section. The photon survival probability saturates at a value

$$
\mathcal{P}(z)=1-2 \chi^{2},
$$

where $\chi$ is the kinetic mixing between photons and hidden photons. The interested reader can find further details about these arguments in [64]. The value of $\chi$ is usually restricted to be smaller than $10^{-3}$ since it has a radiative origin, so there is no foreseeable constraint on this scenario from cosmic opacity. Therefore, we must focus our attention on the pure MCP scenario.

Figure 6 (left) shows 1 and $2-\sigma$ joint confidence levels in the $\kappa-\Omega_{m}$ plane, again for SN data only (dark blue contours) and for the combined $\mathrm{SN}+H(z)$ data set (solid line contours). In this case, SN data alone allow a zero cosmological constant in the presence of MCPs with a value $\kappa \sim 2.3 \times 10^{-4}$ as suggested in [10]. However, the inclusion of $H(z)$ data rules out this possibility and sets a strong bound

$$
\kappa<5 \times 10^{-5} \mathrm{Mpc}^{-1}(2-\sigma),
$$

which, translated into MCP parameters, allows us to constrain the region in parameter space shown in figure 6 (right).

\footnotetext{
${ }^{4}$ In the non-adiabatic regime this scaling is not valid.
} 

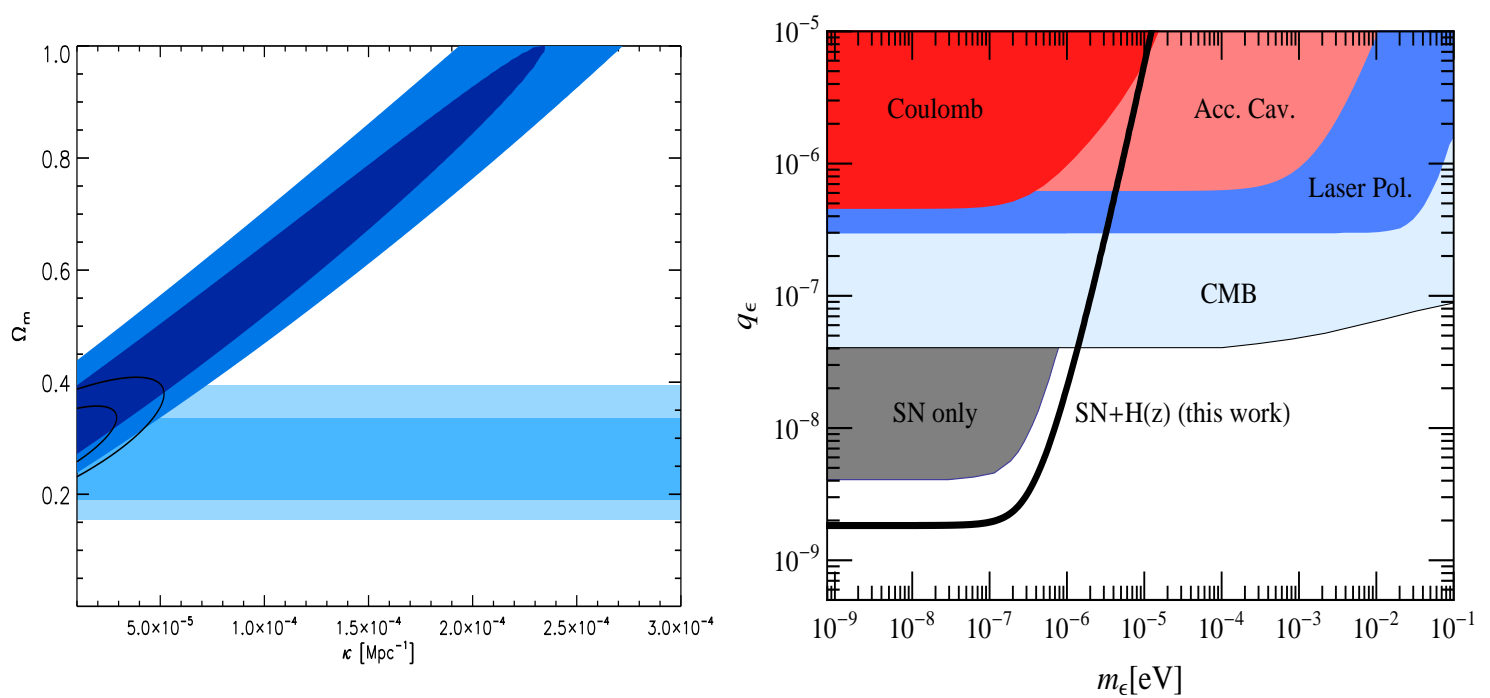

Figure 6. Confidence levels $(68 \%$ and $95 \%)$ on the $\Omega_{m}-\kappa$ plane for the pure MCP model (left panel). Dark blue contours show constraints from SN data only, light blue from $H(z)$ data, and solid line contours from joint $\mathrm{SN}+H(z)$. The right panel shows the corresponding constraints in the minicharge vs MCP mass plane assuming $B=1 \mathrm{nG}$. For comparison we show the previous SN dimming constraints [10] (also for $B=1 \mathrm{nG}$ ), CMB bounds [61] and the most sensitive purely laboratory experiments, light polarization [65], tests of the Coulomb's law [66] and accelerator cavities [67].

\section{Forecasts for future baryon acoustic oscillations and Supernovae surveys}

So far we have investigated constraints on cosmic opacity - and also their implications for models which violate photon number conservation - that are imposed from current data, namely from direct measurements of cosmic expansion $H(z)$ using cosmic chronometers combined with Type Ia Supernova data (in particular the SCP Union 2008 compilation). However, new and more accurate data for $H(z)$ (as well as $d_{A}(z)$, the angular diameter distance) will be available through ongoing and future Baryon acoustic oscillations (BAO) surveys. In this section, we show forecasted constraints for cosmic opacity and the related models of sections 3-4, that can be achieved by combining Supernova and $H(z)$ data from future spectroscopic BAO surveys. We focus in particular on two BAO missions, namely the Baryon Oscillation Spectroscopic Survey (BOSS) and EUCLID. Finally, we also consider forecast constraints from proposed SN missions, in particular combining EUCLID and SNAP.

BOSS [68] is part of the SDSS-III survey and is scheduled to operate over the period 2009-2014. Using the $2.5 \mathrm{~m}$ SDSS telescope, it will measure redshifts of 1.5 million luminous galaxies in the range $0.1<z<0.7$ (as well as Ly $\alpha$ absorption towards 160,000 high-redshift quasars at about $z \simeq 2.5$ ), covering $\simeq 10,000 \mathrm{deg}^{2}$ of high-latitude sky. The forecast precision for $H(z)$ is $1.8 \%, 1.7 \%$ and $1.2 \%$ in redshifts bins centered at $z=0.35,0.6$ and 2.5 respectively. On the other hand, EUCLID - proposed to ESA's Cosmic Visions programme - aims for lunch around 2018. A combination of the earlier SPACE [69] and DUNE [70] missions, EUCLID would provide around 150 million redshifts in the range $z<2$, covering about $30,000 \mathrm{deg}^{2}$. Figure 7 shows forecast errors around the WMAP7 $\Lambda C D M$ model for both BOSS and EUCLID. Also shown for comparison are the current $H(z)$ "cosmic chronometers" data used above. 


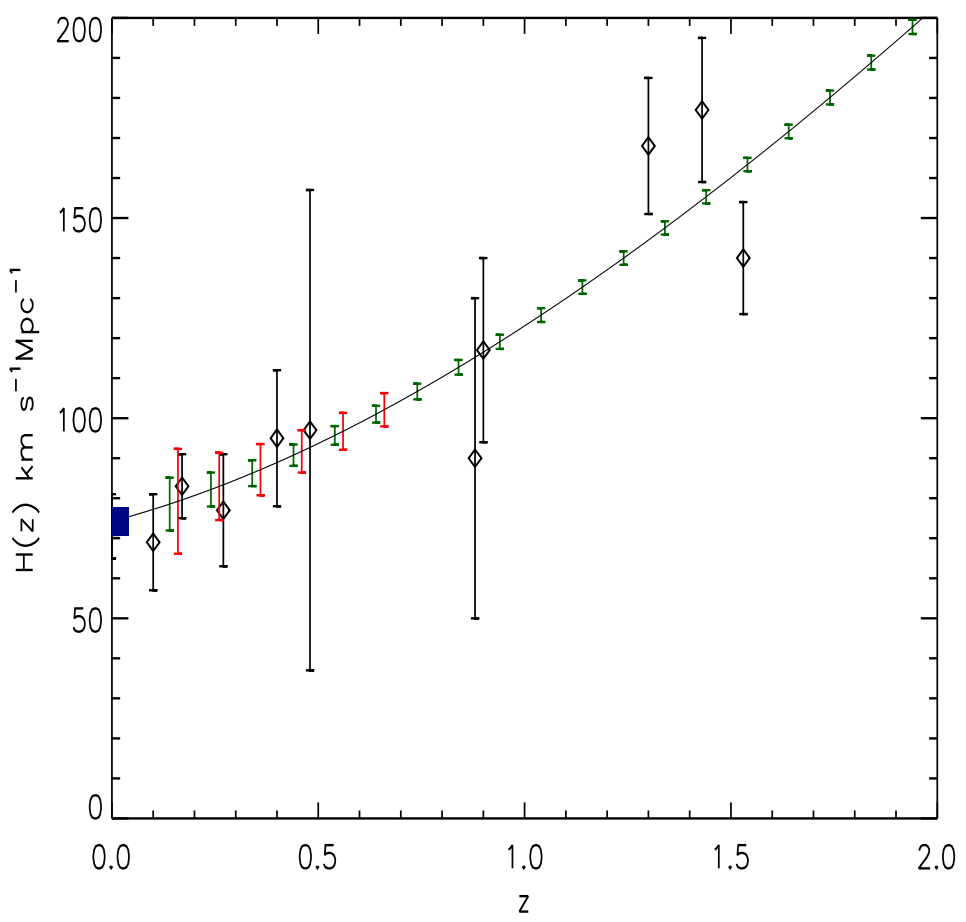

Figure 7. Forecasted error-bars on $H(z)$ for BOSS (red) and EUCLID (green) compared to current $H(z)$ data from differential ageing of galaxies (black). Also shown in the blue rectangle is the Riess et al. determination of $H_{0}$ [24].

We use the code developed by Seo \& Eisenstein [71] to estimate the errors in radial distances achievable by using BAOs as a standard ruler. Figure 8 shows our forecasted constraints on the parameter $\epsilon$ of section 2, using the current type Ia SN data (Union 2008) in combination with modelled BAO data with forecasted errors for both BOSS and EUCLID. ${ }^{5}$ Note that although BOSS will achieve much smaller error bars than those of current $H(z)$ data (cf figure 7), it will span a much narrower redshift range, so it will in fact provide somewhat weaker constraints than the current $H(z)$ "chronometers" data. To make a more direct comparison we have also shown the corresponding constraints obtained by restricting the current $H(z)$ data in the narrower redshift range available to BOSS (thin solid line labeled "chronometer (low z)"). On the other hand, significant improvement of these constraints will be achieved by EUCLID.

Similarly, in figures $9 \& 10$ we show forecasted constraints for the simple ALP model of section $3(A=2 / 3)$ and for chameleons with $A \in[2 / 3,4 / 3]$ (section 3 ). For ALPs, EUCLID will provide significant improvement, notably by a factor of 2-3 on the constraints on $P / L$ in the weak mixing regime of figure 9 . As the probability of mixing is inversely proportional to the square of the energy scale of the ALP coupling, this will result in a modest improvement of the bounds on M by factor of order unity. Finally, forecast constraints for MCPs (section 4) are displayed in figure 11, where, again, EUCLID will improve constraints on the parameter

\footnotetext{
${ }^{5}$ We will consider forecasted constraints combining planned SN missions' data as well at the end of this section.
} 


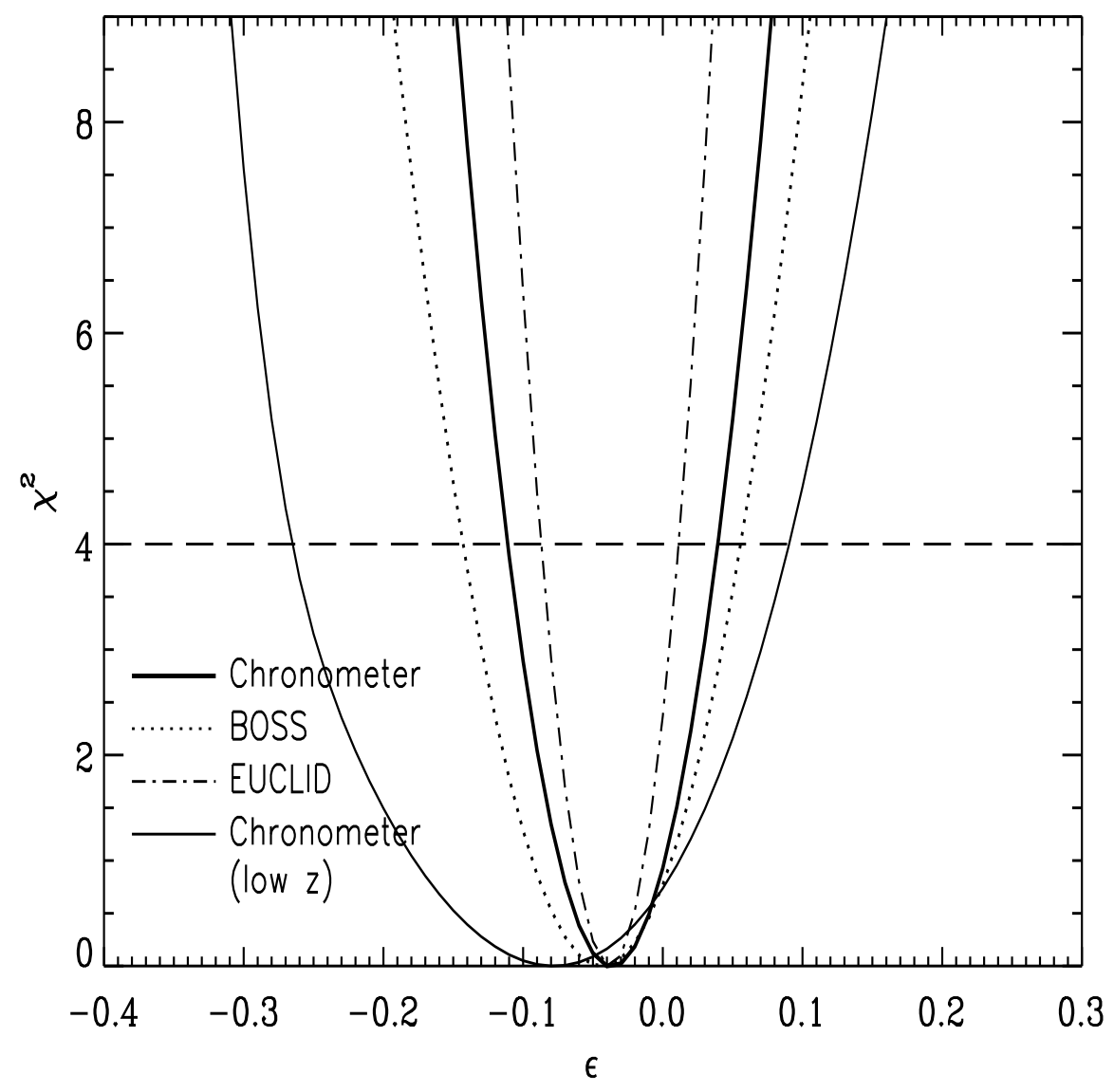

Figure 8. Forecasted constraints on the opacity parameter $\epsilon$ of section 2 for BOSS and EUCLID, combined with current SN data. Also shown are current constraints from $H(z)$ "chronometer" data (again joint with SN). The small shift towards negative $\epsilon$ is due to the fact that actual SN data are being used.

$\kappa$ by a factor of 2-3. As $\kappa \sim q_{\epsilon}^{8 / 3}$, this results in a modest, order unity improvement of the constraints on the charge of any exotic MCP.

So far we have considered the effect that future BAO data will have on the constraints of sections 2-4, when combined with current SN data, and in particular the SCP Union 2008 compilation. We showed that EUCLID will lead to a significant improvement of these constraints (figures 8-11), while the narrower redshift range of BOSS renders it comparable to current $H(z)$ measurements for constraining these models. We end this section by considering the effect that proposed SN surveys data will have on these constraints, in particular forecasted constraints for SNAP (or dark energy task force stage IV SNe mission) [72] combined with EUCLID. Figure 12 summarises these constraints for all models considered above. Our forecasted constraints, shown in orange scale, appear on top of the corresponding joint $\mathrm{SN}+H(z)$ constraints from current data, see sections 3 and 4 . SN data from these proposed surveys will lead to notable improvement of these constraints, for example, by nearly an order of magnitude in the parameter $\epsilon$ described above. As the figure shows, for the models 


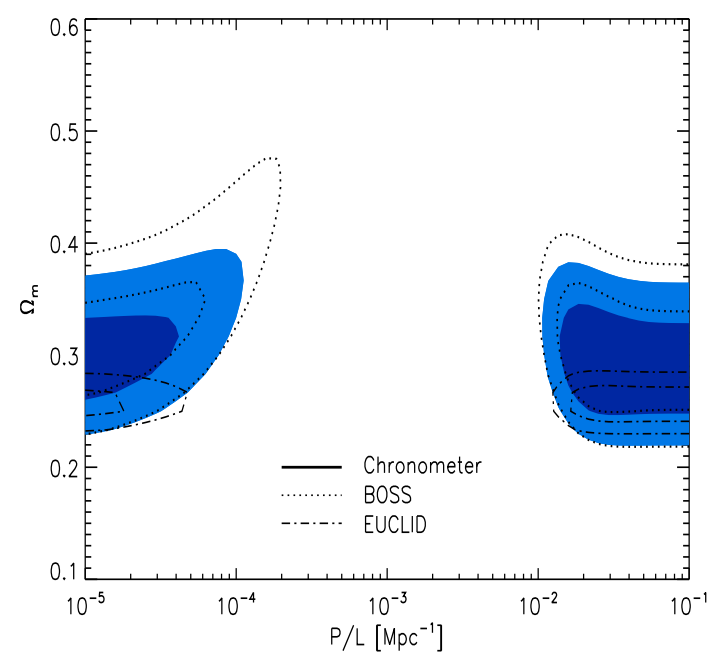

Figure 9. The joint constraints of figure 3 for the simple ALP model of section 3 with $A=2 / 3$, shown together with the corresponding forecast constraints from BOSS and EUCLID combined with current SN data.
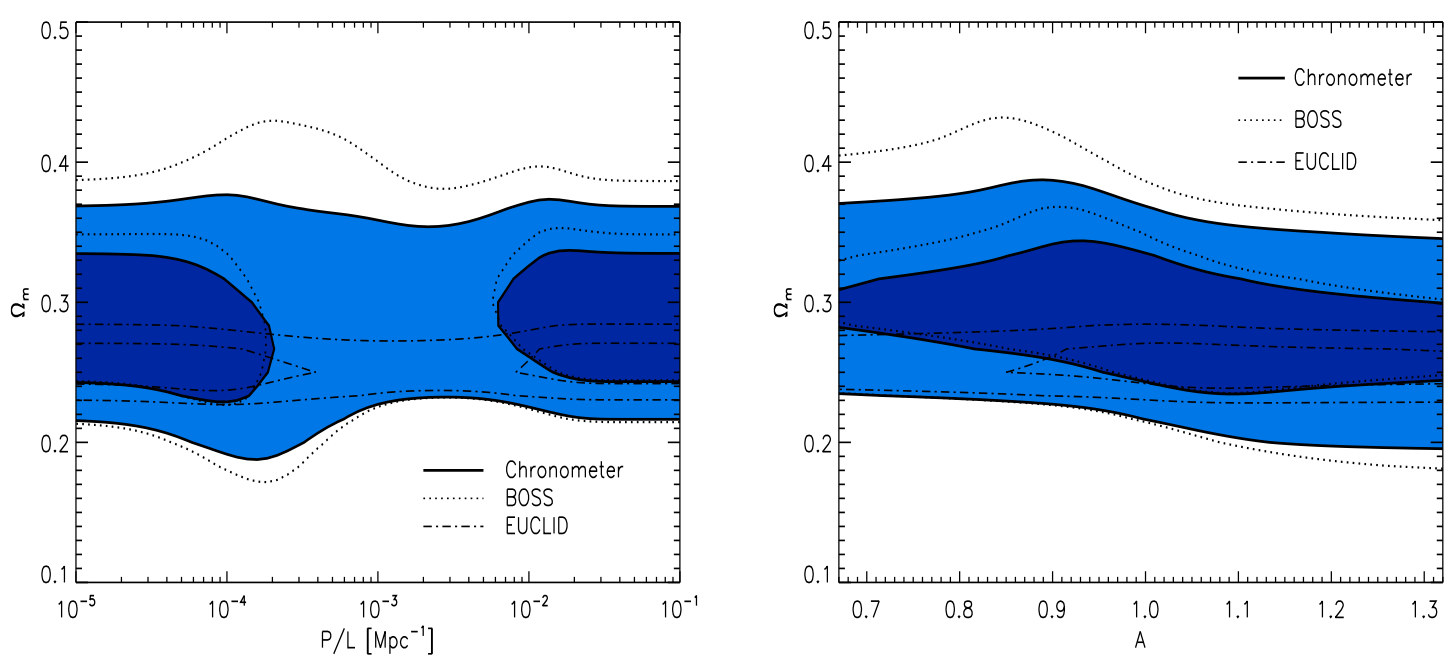

Figure 10. The joint constraints of figure 5 for chameleons with $2 / 3<A<4 / 3$ ), together with the corresponding forecast constraints from BOSS and EUCLID joint with current SN data.

considered above, this will correspond to an improvement of a factor of up to few on the strength of the coupling of ALPs to photons, and on the charge of MCPs.

\section{Conclusions}

If new particles from physics beyond the standard model couple to photons then the propagation of light may be altered. In this paper we have focused on two scenarios for exotic 


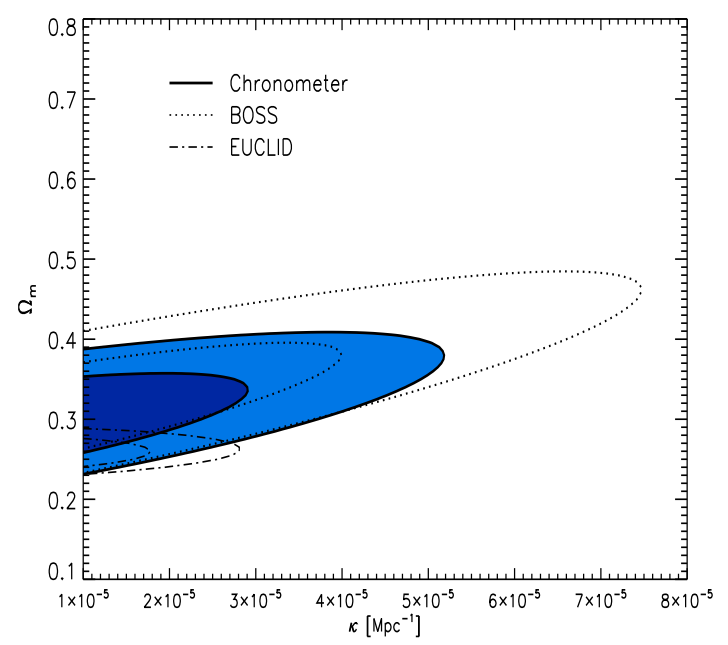

Figure 11. Constraints of figure 6 for MCPs, compared to forecasted constraints from BOSS and EUCLID, combined with current SN data.

particles which can significantly modify the propagation of photons as they pass through magnetic fields. Measurements of cosmic opacity are a strong tool to constrain such scenarios, as interactions between photons and exotic particles in the magnetic fields of the intergalactic medium leads to a new source of cosmic opacity. Uniform deviations from cosmic transparency (i.e. opacity) can be constrained through their effects on distance duality, by parameterizing possible deviations from the Etherington relation. The Etherington relation implies that, in a cosmology based on a metric theory of gravity, distance measures are unique: the luminosity distance is $(1+z)^{2}$ times the angular diameter distance. Both luminosity distance and angular diameter distance depend on the Hubble parameter $H(z)$, but this relation is valid in any cosmological background where photons travel on null geodesics and where, crucially, photon number is conserved. We have restricted our attention on violations of the Etherington relation arising from the violation of photon conservation.

We have combined direct measurements of cosmic expansion (from the latest determinations of the Hubble parameter) at redshifts $0<z<2$ and recent SN data yielding the luminosity distance. SN-inferred luminosity distances are affected by violation of photon conservation, but the $H(z)$ measurements we use are not. Assuming an underlying flat $\Lambda$ CDM model, we have placed tight limits on possible deviations from photon-conservation. Photonconservation can be violated by simple astrophysical effects which give uniform attenuation such as grey dust. We have reported updated constraints on this effect.

More exotic sources of photon-conservation violation involve a coupling of photons to particles beyond the standard model of particle physics. We have focused on axion-like particles, new scalar or pseudo scalar fields which couple to the kinetic terms of photons, and mini-charged particles which are hidden sector particles with a tiny electric charge. Photons passing through intergalactic magnetic fields may be lost by pair production of light minicharged particles. If the mixing between axion-like particles and photons is significant, then interactions in the intergalactic magnetic fields will also lead to a loss of photons due to conversion into ALPs. However if the coupling between photons and ALPs is sufficiently 

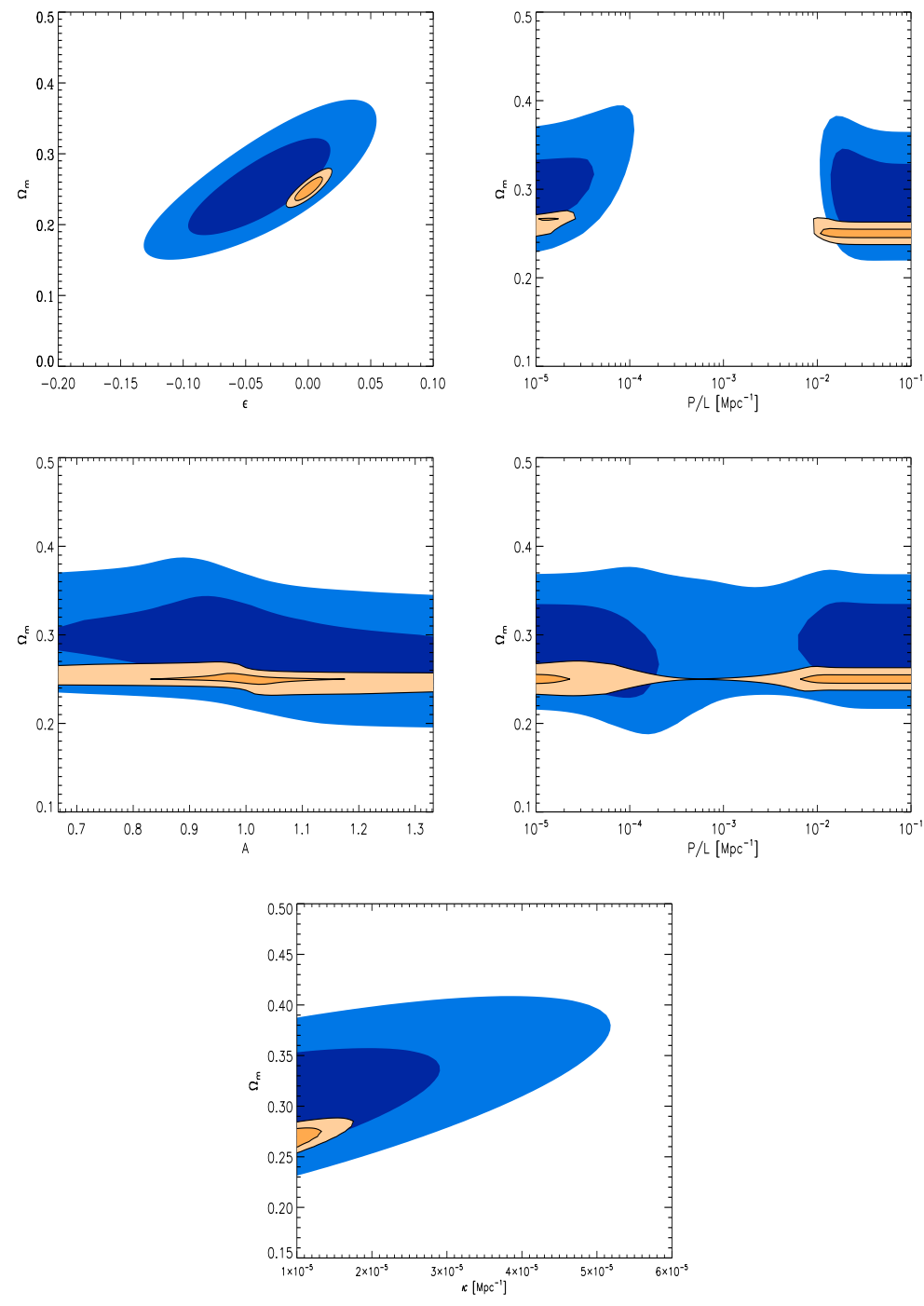

Figure 12. Forecast constraints from joint SNAP+EUCLID (orange scale), shown together with the corresponding constraints from current data, namely SN (Union08) joint with chronometer $H(z)$ (blue scale). Dark and light contours correspond to 1- and 2- $\sigma$ respectively. From top left to bottom right: constraints on the opacity parameter $\epsilon$ (section 2), parameter $P / L$ for the simple ALP model of section 3, parameters $A \& P / L$ for chameleons (section 3 ), and parameter $\kappa$ for MCPs (section 4 ).

strong, one-third of any initial flux will be converted into ALPs, and two-thirds into photons, resulting in a redshift-independent dimming of supernovae which we cannot constrain or exclude with cosmic opacity bounds.

It is also possible to search for the effects of new physics on the propagation of photons in the laboratory. In such experiments a laser beam is shone through a vacuum tube placed in a magnetic field. Then, changes in the polarization of the light beam due to interactions with exotic particles can be constrained. In addition, more exotic effects, such as the possibility of 'shining light through walls' due to photons converting into exotic particles and then back into photons, can be searched for and constrained with experiment. A more detailed description 

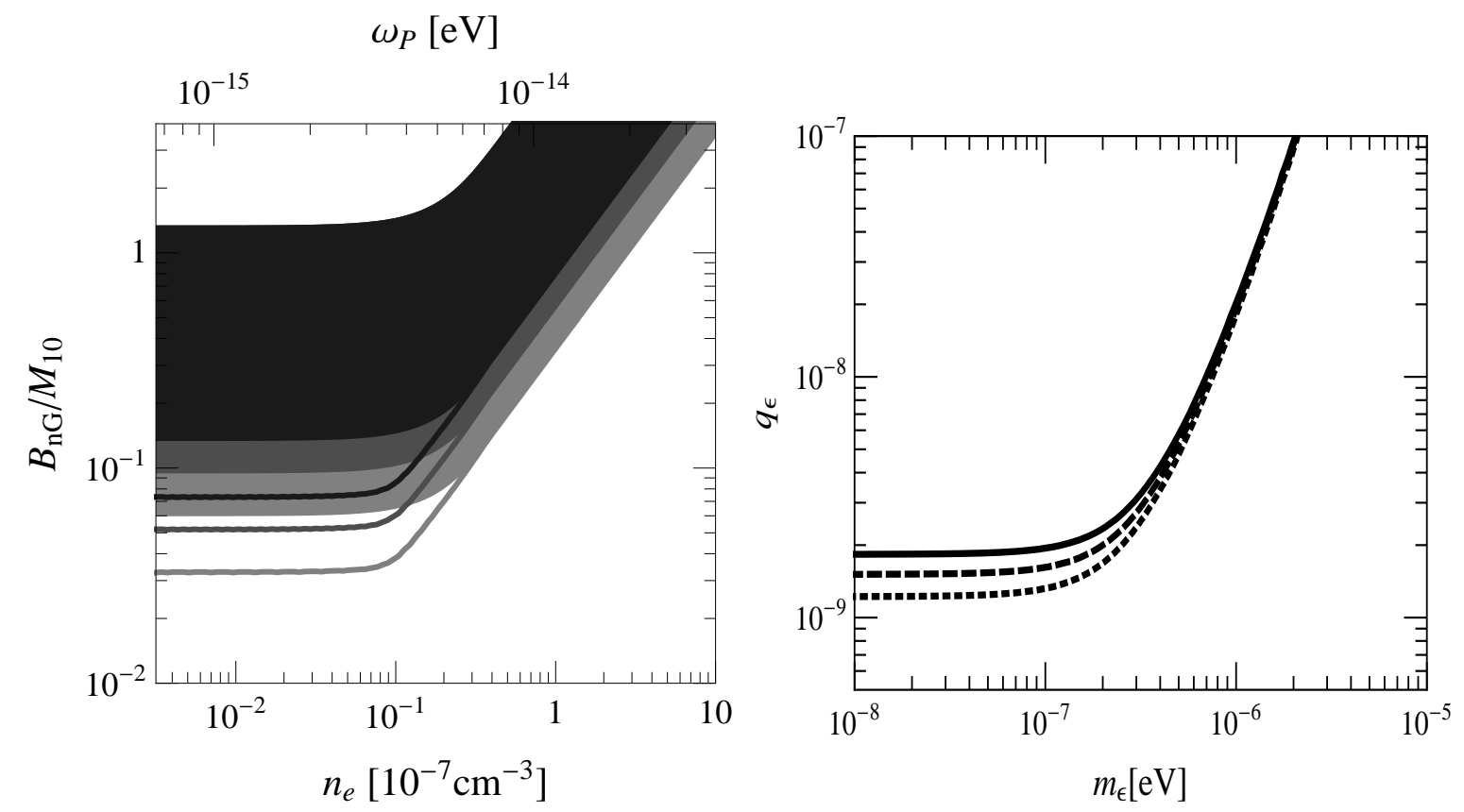

Figure 13. Prospects of the future bounds on ALPs (left) and MCPs (right). ALPs: The dark region corresponds to the current bounds while grey and light grey regions cover the possible future bounds using EUCLID and EUCLID+SNAP data. Taking into account the redshift dependence of the background improves these bounds in the region covered by the lines. MCPs: Current bounds (solid) and prospects from EUCLID (dashed) and EUCLID+SNAP (dotted).

of such experiments, and the constraints they impose on the models we have considered can be found in [5]. In the regions of parameter space of these models where the transparency of the universe imposes constraints, the bounds from the analysis given in the proceeding sections are at least two orders of magnitude better than what can currently be achieved with laboratory experiments.

The improved measurement of the cosmic opacity found here leads to improved bounds on these exotic physics scenarios which are summarised in figure 13. Future measurements of baryon acoustic oscillations, and an increase in the number of observations of high redshift supernovae will lead to further improvements in the constraints on physics beyond the standard model.

\section{Acknowledgments}

The work of AA is funded by a CTC Postdoctoral Fellowship. AA would like to thank the Institute of Cosmos Sciences, University of Barcelona, for hospitality and support. CB is supported by the German Science Foundation (DFG) under the Collaborative Research Centre (SFB) 676. JR acknowledges support from the DFG cluster of excellence EXC 153 Origin and Structure of the Universe. LV is supported by FP7-PEOPLE-2007-4-3 IRG $\mathrm{n}$ 202182 and FP7 IDEAS-Phys.LSS.240117. RJ is supported by FP7-PEOPLE-2007-4-3 IRG. LV and RJ are also supported by MICINN grant AYA2008-03531. 


\section{A Summary of key formulae}

Here we summarise the relevant equations describing how the luminosity distance measures are affected by the presence of particles beyond the standard model that couple to photons: axion-like particles (ALP), chameleons and mini-charged particles (MCP).

The presence of ALPs will have an impact on observations of supernovae if they are observed through magnetic fields. The strength and spatial structure of the inter-galactic magnetic fields is highly unconstrained and so they are left as parameters. The luminosity distance to $\mathrm{SNe}$ is modified by the redshift-dependent probability of photon survival $\mathcal{P}(z)$ :

$$
d_{L}(z) \rightarrow d_{L}(z) / \sqrt{\mathcal{P}(z)} .
$$

There are two physical limits in which $\mathcal{P}(z)$ can be analytically calculated, namely the incoherent and coherent regimes. In the incoherent regime one has:

$$
\mathcal{P}(z)=A+(1-A) \exp \left(-\frac{3}{2} \frac{y(z)}{L} P\right),
$$

where $L$ is the size of magnetic domains, $P$ the probability of photon to ALP conversion, and $y(z)$ the comoving distance to the source. In the coherent regime, one can take into account additional effects due to evolution of the background magnetic fields with redshift. In this case the probability of photon survival reads:

$$
\mathcal{P}(z)=A+(1-A) \exp \left(-\frac{P}{H_{0} L} \frac{H(z)-H_{0}}{\Omega_{m} H_{0}}\right),
$$

where $H_{0}$ and $\Omega_{m}$ are the Hubble constant and matter density parameter (at the present epoch) respectively, and $H(z)$ is the Hubble parameter at redshift $z$. For the simplest ALP models, $A=2 / 3$ in the above equations, while for chameleons these equations hold with $A \neq 2 / 3$.

The existence of low-mass MCPs can also have a tremendous impact on photon propagation over cosmological distances. Photons from a given source can for instance pair produce MCPs, even without the need for a (CMB) photon, in the presence of an inter-galactic magnetic field. The MCP pair production process damps the photon flux, so, again, the luminosity distance to SNe is modified by a redshift-dependent probability of photon survival, $\mathcal{P}(z)$. In this case one has:

$$
\mathcal{P}(z)=\exp (-\kappa y(z)),
$$

where $\kappa$ is the photon to MCP transition rate and is given by

$$
\kappa=\frac{e^{8 / 3}}{4 \Gamma\left(\frac{1}{6}\right) \Gamma\left(\frac{13}{6}\right)}\left(\frac{2 B^{2} q_{\epsilon}^{8}}{3 \omega}\right)^{1 / 3} f .
$$

Here, $B$ denotes the magnetic field strength, $\omega$ is the photon energy, $q_{\epsilon}$ is the MCP electric charge in units of the electron charge $e$, and $f$ is an order unity factor which depends on the nature of the $\mathrm{MCP}$ and the photon polarization with respect to the magnetic field. $\Gamma$ denotes the usual $\Gamma$-function. 


\section{References}

[1] J.M.H. Etherington, On the definition of distance in general relativity, Phil. Mag. 15 (1933) 761.

[2] B. Ménard, D. Nestor, D. Turnshek, A. Quider, G. Richards, D. Chelouche and S. Rao, Lensing, reddening and extinction effects of $M g$ ii absorbers from $z=0.4$ to 2 , Mon Not. Roy. Astron. Soc. 385 (2008) 1053.

[3] J. Bovy, D.W. Hogg and J. Moustakas, The Transparency of Galaxy Clusters, Astrophys. J. 688 (2008) 198 [arXiv:0805.1200] [SPIRES].

[4] A.N. Aguirre, Dust Versus Cosmic Acceleration, Astrophys. J 512 (1999) L19 [astro-ph/9811316] [SPIRES].

[5] J. Jaeckel and A. Ringwald, The Low-Energy Frontier of Particle Physics, arXiv:1002.0329 [SPIRES].

[6] C. Csáki, N. Kaloper and J. Terning, Dimming supernovae without cosmic acceleration, Phys. Rev. Lett. 88 (2002) 161302 [hep-ph/0111311] [SPIRES].

[7] E. Mörtsell, L. Bergström and A. Goobar, Photon axion oscillations and type-IA supernovae, Phys. Rev. D 66 (2002) 047702 [astro-ph/0202153] [SPIRES].

[8] A. Mirizzi, G.G. Raffelt and P.D. Serpico, Photon axion conversion in intergalactic magnetic fields and cosmological consequences, Lect. Notes Phys. 741 (2008) 115 [astro-ph/0607415] [SPIRES].

[9] C. Burrage, Supernova Brightening from Chameleon-Photon Mixing, Phys. Rev. D 77 (2008) 043009 [arXiv:0711.2966] [SPIRES].

[10] M. Ahlers, The Hubble diagram as a probe of mini-charged particles, Phys. Rev. D 80 (2009) 023513 [arXiv:0904.0998] [SPIRES].

[11] B.A. Bassett, Cosmic acceleration vs axion photon mixing, Astrophys. J. 607 (2004) 661 [astro-ph/0311495] [SPIRES].

[12] B.A. Bassett and M. Kunz, Is cosmic distance-duality violated?, Phys. Rev. D 69 (2004) 101305 [astro-ph/0312443] [SPIRES].

[13] Y.-S. Song and W. Hu, Constraints on supernovae dimming from photon - pseudo scalar coupling, Phys. Rev. D 73 (2006) 023003 [astro-ph/0508002] [SPIRES].

[14] S. More, J. Bovy and D.W. Hogg, Cosmic transparency: A test with the baryon acoustic feature and type-IA supernovae, Astrophys. J. 696 (2009) 1727 [arXiv:0810.5553] [SPIRES].

[15] A. Avgoustidis, L. Verde and R. Jimenez, Consistency among distance measurements: transparency, BAO scale and accelerated expansion, JCAP 06 (2009) 012 [arXiv:0902.2006] [SPIRES].

[16] M. Kowalski et al., Improved Cosmological Constraints from New, Old, and Combined Supernova Data Sets, Astrophys. J. 686 (2008) 749 [arXiv:0804.4142] [SPIRES].

[17] R. Jimenez, L. Verde, T. Treu, and D. Stern, Constraints on the equation of state of dark energy and the Hubble constant from stellar ages and the CMB, Astrophys. J. 593 (2003) 622 [astro-ph/0302560] [SPIRES].

[18] J. Simon, L. Verde and R. Jimenez, Constraints on the redshift dependence of the dark energy potential, Phys. Rev. D 71 (2005) 123001 [astro-ph/0412269] [SPIRES].

[19] V.A. Kostelecky and N. Russell, Data Tables for Lorentz and CPT Violation, arXiv:0801.0287 [SPIRES].

[20] J.-P. Uzan, The acceleration of the universe and the physics behind it, Gen. Rel. Grav. 39 (2007) 307 [astro-ph/0605313] [SPIRES]. 
[21] R. Lazkoz, S. Nesseris and L. Perivolaropoulos, Comparison of Standard Ruler and Standard Candle constraints on Dark Energy Models, JCAP 07 (2008) 012 [arXiv:0712.1232] [SPIRES].

[22] W.J. Percival et al., Measuring the Baryon Acoustic Oscillation scale using the SDSS and 2dFGRS, Mon. Not. Roy. Astron. Soc. 381 (2007) 1053 [arXiv:0705.3323] [SPIRES].

[23] D. Stern, R. Jimenez, L. Verde, M. Kamionkowski and S.A. Stanford, Cosmic Chronometers: Constraining the Equation of State of Dark Energy. I: H(z) Measurements, JCAP 02 (2010) 008 [arXiv: 0907.3149] [SPIRES].

[24] A.G. Riess et al., A Redetermination of the Hubble Constant with the Hubble Space Telescope from a Differential Distance Ladder, Astrophys. J. 699 (2009) 539 [arXiv:0905.0695] [SPIRES].

[25] W.L. Freedman et al., Final Results from the Hubble Space Telescope Key Project to Measure the Hubble Constant, Astrophys. J. 553 (2001) 47 [astro-ph/0012376] [SPIRES].

[26] R. Kessler et al., First-Year Sloan Digital Sky Survey-II Supernova Results: Hubble Diagram and Cosmological Parameters, Astrophys. J. Suppl. 185 (2009) 32 [arXiv:0908.4274] [SPIRES].

[27] H. Lampeitl et al., First-year Sloan Digital Sky Survey-II (SDSS-II) supernova results: consistency and constraints with other intermediate-redshift datasets, Mon. Not. Roy. Astron. Soc. 401 (2009) 2331 [arXiv:0910.2193] [SPIRES].

[28] R.D. Peccei and H.R. Quinn, CP Conservation in the Presence of Instantons, Phys. Rev. Lett. 38 (1977) 1440 [SPIRES].

[29] P. Svrček and E. Witten, Axions in string theory, JHEP 06 (2006) 051 [hep-th/0605206] [SPIRES].

[30] A.A. Anselm and N.G. Uraltsev, A Second Massless Axion?, Phys. Lett. B 114 (1982) 39 [SPIRES].

[31] P. Brax, C. Burrage, A.-C. Davis, D. Seery and A. Weltman, Higgs production as a probe of Chameleon Dark Energy, Phys. Rev. D 81 (2010) 103524 [arXiv:0911.1267] [SPIRES].

[32] J. Khoury and A. Weltman, Chameleon Fields: Awaiting Surprises for Tests of Gravity in Space, Phys. Rev. Lett. 93 (2004) 171104 [astro-ph/0309300] [SPIRES].

[33] J. Khoury and A. Weltman, Chameleon Cosmology, Phys. Rev. D 69 (2004) 044026 [astro-ph/0309411] [SPIRES].

[34] C. Burrage, A.-C. Davis and D.J. Shaw, Detecting Chameleons: The Astronomical Polarization Produced by Chameleon-like Scalar Fields, Phys. Rev. D 79 (2009) 044028 [arXiv:0809.1763] [SPIRES].

[35] P. Brax, C. van de Bruck, A.-C. Davis, J. Khoury and A. Weltman, Detecting dark energy in orbit: The cosmological chameleon, Phys. Rev. D 70 (2004) 123518 [astro-ph/0408415] [SPIRES].

[36] P. Brax, C. van de Bruck and A.-C. Davis, Compatibility of the chameleon-field model with fifth- force experiments, cosmology and PVLAS and CAST results, Phys. Rev. Lett. 99 (2007) 121103 [hep-ph/0703243] [SPIRES].

[37] G. Raffelt and L. Stodolsky, Mixing of the Photon with Low Mass Particles, Phys. Rev. D 37 (1988) 1237 [SPIRES].

[38] J.D. Barrow, P.G. Ferreira and J. Silk, Constraints on a Primordial Magnetic Field, Phys. Rev. Lett. 78 (1997) 3610 [astro-ph/9701063] [SPIRES].

[39] P. Blasi, S. Burles and A.V. Olinto, Cosmological Magnetic Fields Limits in an Inhomogeneous Universe, Astrophys. J. 514 (1999) L79 [astro-ph/9812487] [SPIRES].

[40] P.P. Kronberg, Extragalactic magnetic fields, Rept. Prog. Phys. 57 (1994) 325. 
[41] A.-C. Davis, C.A.O. Schelpe and D.J. Shaw, The Effect of a Chameleon Scalar Field on the Cosmic Microwave Background, Phys. Rev. D 80 (2009) 064016 [arXiv:0907.2672] [SPIRES].

[42] C.A.O. Schelpe, Chameleon-Photon Mixing in a Primordial Magnetic Field, Phys. Rev. D 82 (2010) 044033 [arXiv: 1003.0232] [SPIRES].

[43] P.J.E. Peebles, Principles of Physical Cosmology, Princeton Univ. Press, Princeton U.S.A. (1993).

[44] C. Csáki, N. Kaloper and J. Terning, Effects of the intergalactic plasma on supernova dimming via photon axion oscillations, Phys. Lett. B 535 (2002) 33 [hep-ph/0112212] [SPIRES].

[45] C. Deffayet, D. Harari, J.-P. Uzan and M. Zaldarriaga, Dimming of supernovae by photon pseudoscalar conversion and the intergalactic plasma, Phys. Rev. D 66 (2002) 043517 [hep-ph/0112118] [SPIRES].

[46] Y. Grossman, S. Roy and J. Zupan, Effects of initial axion production and photon axion oscillation on type-IA supernova dimming, Phys. Lett. B 543 (2002) 23 [hep-ph/0204216] [SPIRES].

[47] A. Mirizzi and D. Montanino, Stochastic conversions of TeV photons into axion-like particles in extragalactic magnetic fields, JCAP 12 (2009) 004 [arXiv:0911.0015] [SPIRES].

[48] C. Burrage, A.-C. Davis and D.J. Shaw, Active Galactic Nuclei Shed Light on Axion-likeParticles, Phys. Rev. Lett. 102 (2009) 201101 [arXiv:0902. 2320] [SPIRES].

[49] A. Mirizzi, G.G. Raffelt and P.D. Serpico, Photon-axion conversion as a mechanism for supernova dimming: Limits from CMB spectral distortion, Phys. Rev. D 72 (2005) 023501 [astro-ph/0506078] [SPIRES].

[50] L. Ostman and E. Mortsell, Limiting the dimming of distant type-IA supernovae, JCAP 02 (2005) 005 [astro-ph/0410501] [SPIRES].

[51] E. Mörtsell and A. Goobar, Constraints on intergalactic dust from quasar colours, JCAP 09 (2003) 009 [astro-ph/0308046] [SPIRES].

[52] E. Mörtsell and A. Goobar, Constraining photon-axion oscillations using quasar spectra, JCAP 04 (2003) 003 [SPIRES].

[53] B. Holdom, Two U(1)'s and Epsilon Charge Shifts, Phys. Lett. B 166 (1986) 196 [SPIRES].

[54] B. Batell and T. Gherghetta, Localized U(1) gauge fields, millicharged particles and holography, Phys. Rev. D 73 (2006) 045016 [hep-ph/0512356] [SPIRES].

[55] F. Brummer, J. Jaeckel and V.V. Khoze, Magnetic Mixing - Electric Minicharges from Magnetic Monopoles, JHEP 06 (2009) 037 [arXiv:0905.0633] [SPIRES].

[56] S.A. Abel, J. Jaeckel, V.V. Khoze and A. Ringwald, Illuminating the hidden sector of string theory by shining light through a magnetic field, Phys. Lett. B 666 (2008) 66 [hep-ph/0608248] [SPIRES].

[57] S.A. Abel, M.D. Goodsell, J. Jaeckel, V.V. Khoze and A. Ringwald, Kinetic Mixing of the Photon with Hidden U(1)s in String Phenomenology, JHEP 07 (2008) 124 [arXiv:0803.1449] [SPIRES].

[58] K.R. Dienes, C.F. Kolda and J. March-Russell, Kinetic mixing and the supersymmetric gauge hierarchy, Nucl. Phys. B 492 (1997) 104 [hep-ph/9610479] [SPIRES].

[59] S.A. Abel and B.W. Schofield, Brane-antibrane kinetic mixing, millicharged particles and SUSY breaking, Nucl. Phys. B 685 (2004) 150 [hep-th/0311051] [SPIRES].

[60] M. Goodsell, J. Jaeckel, J. Redondo and A. Ringwald, Naturally Light Hidden Photons in LARGE Volume String Compactifications, JHEP 11 (2009) 027 [arXiv:0909.0515] [SPIRES]. 
[61] A. Melchiorri, A. Polosa and A. Strumia, New bounds on millicharged particles from cosmology, Phys. Lett. B 650 (2007) 416 [hep-ph/0703144] [SPIRES].

[62] H. Gies, J. Jaeckel and A. Ringwald, Polarized light propagating in a magnetic field as a probe of millicharged fermions, Phys. Rev. Lett. 97 (2006) 140402 [hep-ph/0607118] [SPIRES].

[63] M. Ahlers, H. Gies, J. Jaeckel, J. Redondo and A. Ringwald, Light from the Hidden Sector, Phys. Rev. D 76 (2007) 115005 [arXiv:0706. 2836] [SPIRES].

[64] C. Burrage, J. Jaeckel, J. Redondo and A. Ringwald, Late time CMB anisotropies constrain mini-charged particles, JCAP 11 (2009) 002 [arXiv: 0909.0649] [SPIRES].

[65] M. Ahlers, H. Gies, J. Jaeckel, J. Redondo and A. Ringwald, Laser experiments explore the hidden sector, Phys. Rev. D 77 (2008) 095001 [arXiv:0711.4991] [SPIRES].

[66] J. Jaeckel, Probing Minicharged Particles with Tests of Coulomb's Law, Phys. Rev. Lett. 103 (2009) 080402 [arXiv:0904.1547] [SPIRES].

[67] H. Gies, J. Jaeckel and A. Ringwald, Accelerator cavities as a probe of millicharged particles, Europhys. Lett. 76 (2006) 794 [hep-ph/0608238] [SPIRES].

[68] With InPUT From the SDSS-III collaboration, D. Schlegel, M. White and D. Eisenstein, The Baryon Oscillation Spectroscopic Survey: Precision measurements of the absolute cosmic distance scale, arXiv:0902.4680 [SPIRES].

[69] A. Cimatti et al., SPACE: the SPectroscopic All-sky Cosmic Explorer, Exper. Astron. 23 (2009) 39 [arXiv:0804.4433] [SPIRES].

[70] A. Refregier, M. Douspis and t.D. collaboration, Summary of the DUNE Mission Concept, arXiv:0807.4036 [SPIRES].

[71] H.-J. Seo and D.J. Eisenstein, Improved forecasts for the baryon acoustic oscillations and cosmological distance scale, Astrophys. J. 665 (2007) 14 [astro-ph/0701079] [SPIRES].

[72] A. Albrecht et al., Report of the Dark Energy Task Force, astro-ph/0609591 [SPIRES]. 